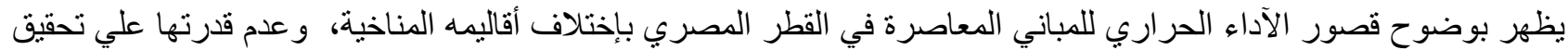

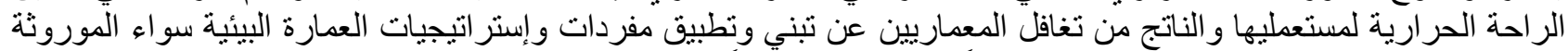

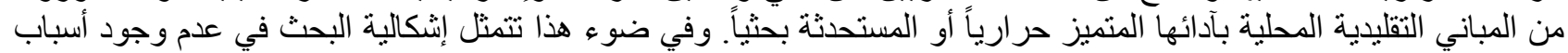

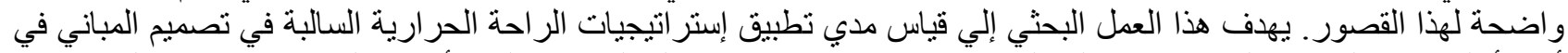

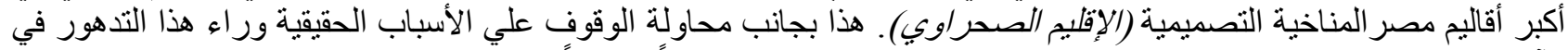

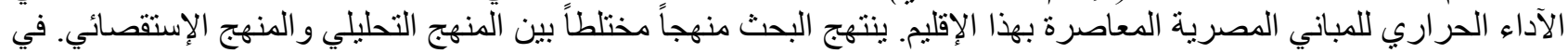

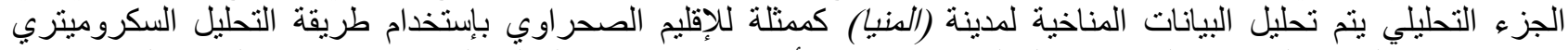

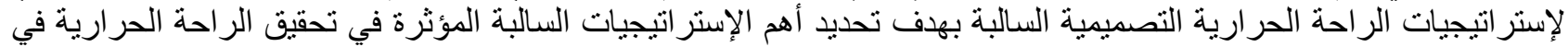

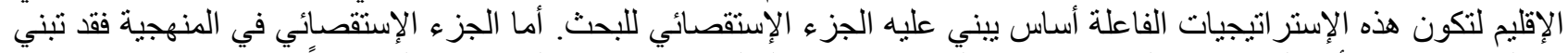

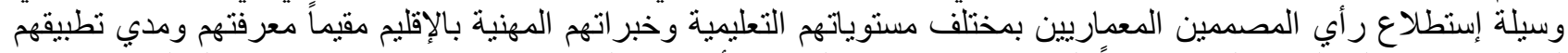

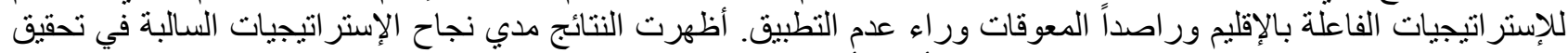

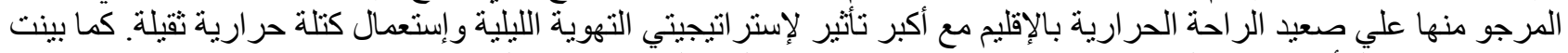

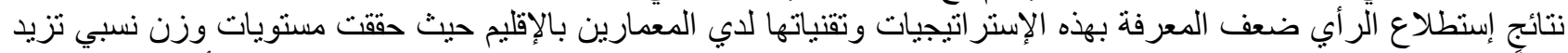

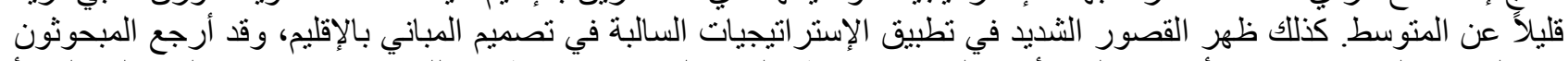

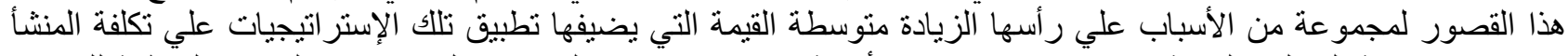

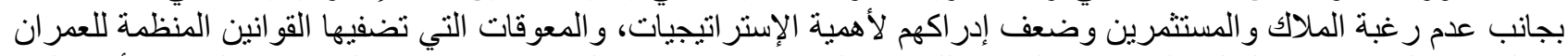

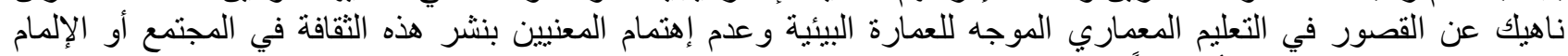

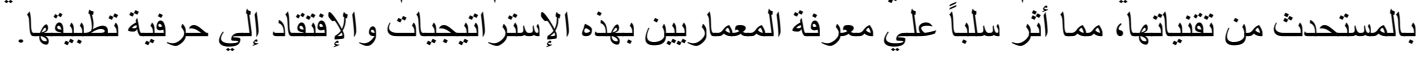

الكلمات المفتاحية: الآداء الحر اري للمباني ـ الراحة الحرارية ـ الإستر اتيجيات السالبة ـ الإقليم الصحر اوي - مصر. 


\title{
Evaluating the application of thermal comfort passive strategies in buildings' design within the Egyptian desert climatic design region
}

\author{
Ashraf A. Abd Al-Raheem* Karima A. M. Attia** Medhat M. A. Osman*** \\ * Architectural professor, Architecture Dept., Faculty of engineering, Minia university, Egypt \\ ** Instructor, Architecture Dept., El-Minya high institute for engineering and technology in New El-Minya, Egypt \\ *** Environmental design assistant prof., Architecture Dept., Faculty of engineering, Minia university, Egypt \\ Corresponding author email: medhat.osman@mu.edu.eg
}

\begin{abstract}
:
The deficiency of the thermal performance of contemporary buildings in Egypt, with its different climatic design regions, lead to poor thermal comfort for buildings' users. This is probably caused by designs that neglect adopting the Environmental techniques and strategies in. The problem is that there are no clear reasons for this deficiency. This research aims to evaluate the application of passive thermal comfort strategies in buildings' design within the largest Egyptian climatic design region, namely (the desert region). In addition, it tries to identify the causes behind the deterioration in the thermal performance of contemporary Egyptian buildings across this region. The research employs a mixed methodology that includes analytical and investigative approaches. In the analytical part, the climatic data for (Minya) city (the representative city of the desert region) are analyzed and the most effective strategies in achieving thermal comfort within the region are identified. These effective strategies are the basis upon which the investigative part of the research is based. As for the investigative part of the methodology, questionnaire technique is adopted to collect data from local architects and experts with various qualifications and professional experiences. They are asked to assess their knowledge with effective passive strategies and their usage in design, as well as, define the constrains behind non-implementation. The results showed that the passive strategies were significantly effective in achieving thermal comfort in the region, with the greatest effect of both; night purge ventilation strategy and using heavy thermal mass strategy. The results also indicated a lack of knowledge of these strategies among local architects with relative weight slightly higher than average level. In addition, there was a significant deficiency in their application of passive strategies in their designs. The respondents referred this deficiency to some reasons. The medium-value increase in building's cost, that these strategies application add, was on the top of these reasons. Other reasons were; the unwillingness of owners / investors along with their poor awareness of these strategies' importance, the obstacles that buildings' laws add, and finally, the shortcomings in architectural education concerned with environmental design. This in turn, negatively affects architects' knowledge of these strategies and causes their weak application in practice.
\end{abstract}

Keywords: Buildings' performance, Thermal comfort, passive strategies, Desert Region, Egypt 
أما علي مستوي المباني التقليدية وتصميمها و وآدائها

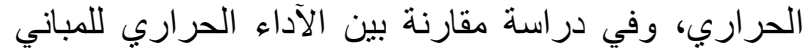

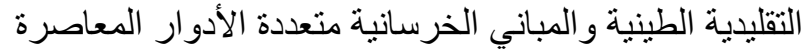

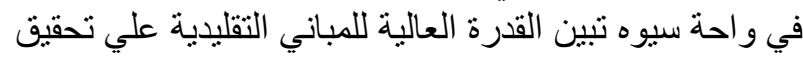

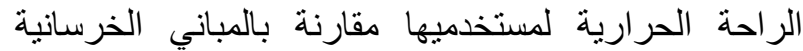

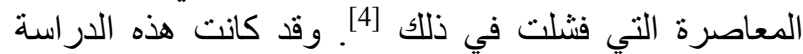

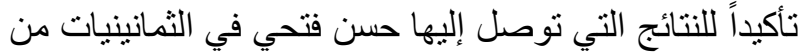

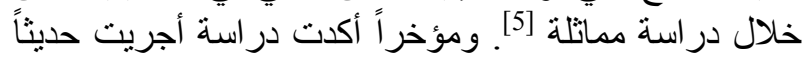

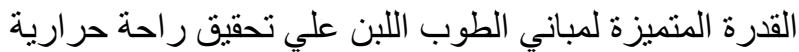

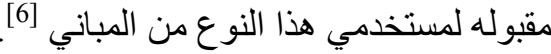

ورغم الأداء الحراري المتميز لهذا النوع من المباني إلا أنه

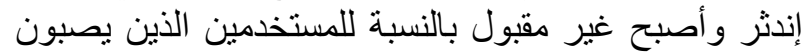

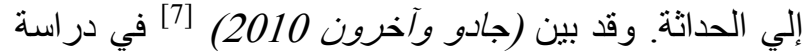

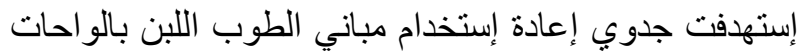

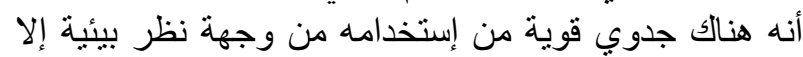

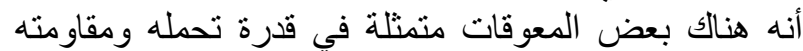

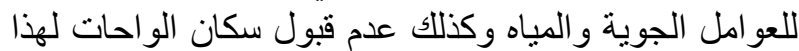

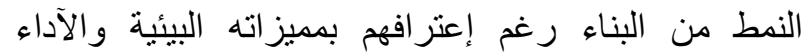

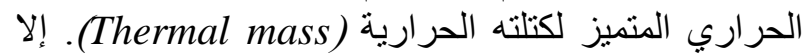

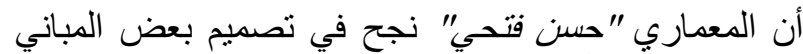

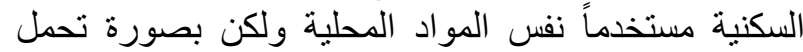

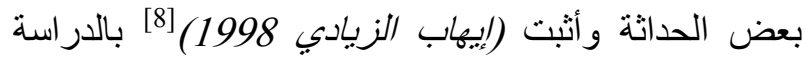

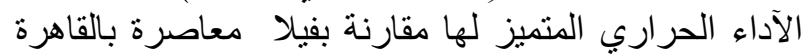
مع قبول المجتمع المحلي لها و إنغالها بأسر الطبقة الغنية لئنية

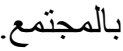

وقد أثتتت الدراسات حرفية إستخدام إستراتيجيات التبريد

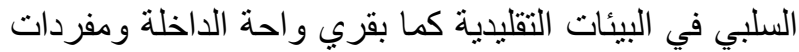

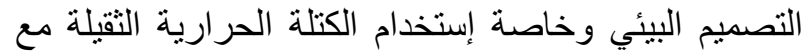

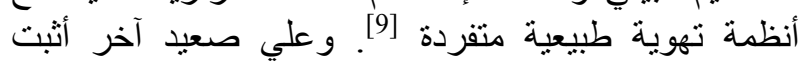

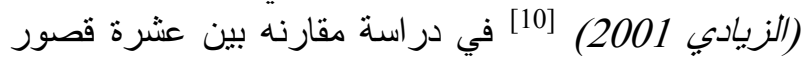
من قصور القاهرة الخديوية التي تم تحويلها لمقرات إدارية إدارية

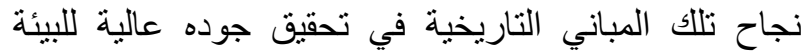

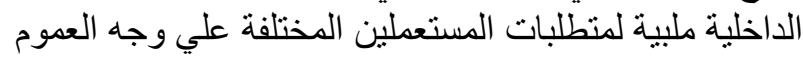

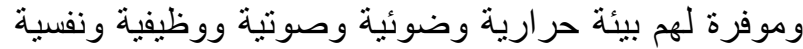

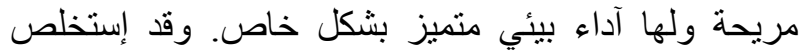

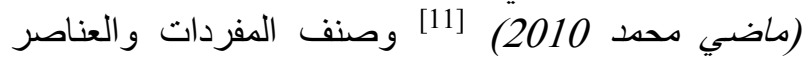

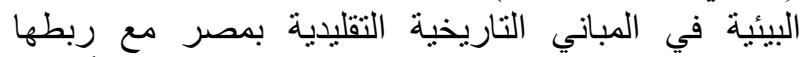
بالإستر اتيجيات السالبة لتحقيق الراحة الحرارية التية مقدماً دليلاً

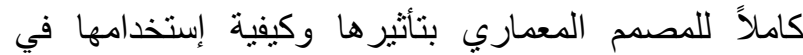

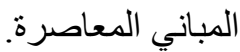

\section{1 ـ 2 الآداء الحراري للمباني المصرية المعاصرة :}

وفي سياق آخر فقد تنوعت الأدبيات و الدراسات التي إهتمت

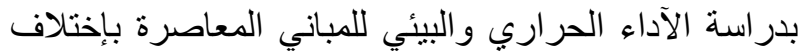

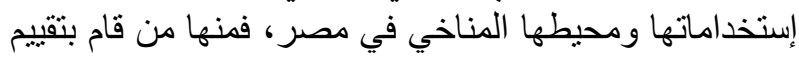

1. المقدمة والخلفية العلمية للاراسة :

علي مدار أكثر من نصف قرن تباينت وإختلفت رؤية

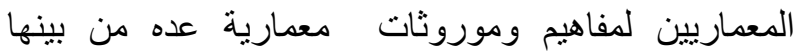

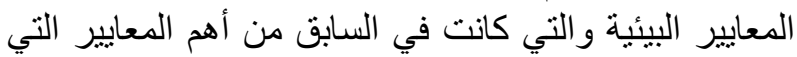

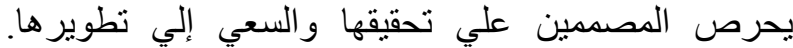

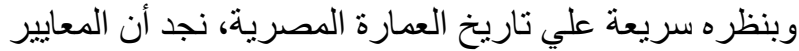

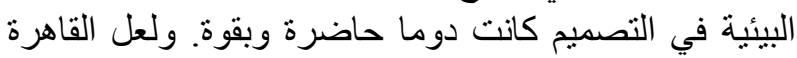

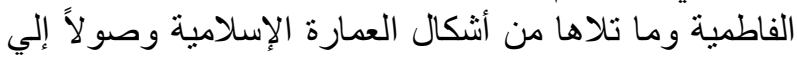

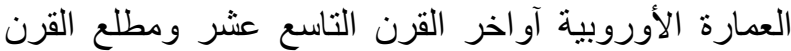
العشرين خير مثال علي الإهتمام الكبير بالقيمة البيئية للمباني التئي

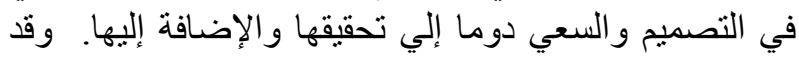

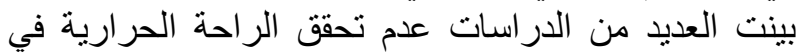

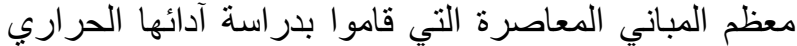

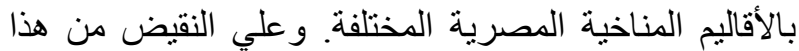

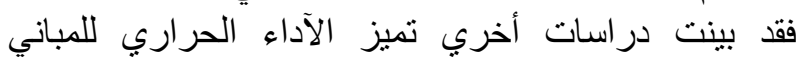

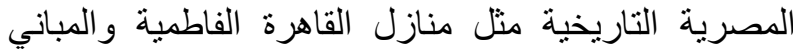

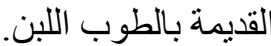

\section{1 ـ 1 الآداع الصراري للمباني المصرية التراثية :}

علي مستوي الفراغات شبه الخاصة والأفنية الداخلية في المباني التقليدية المصرية متمثلة في مباني القاهرة الإسلامية،

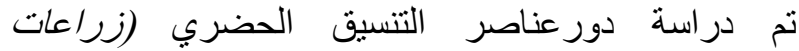
ومسطحات مائية) و الأفنية الداخلية في تحسين الآداء

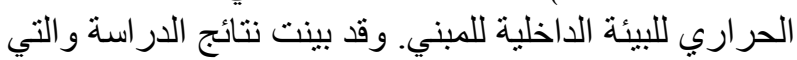
إتخذت بيت السحيمي كأحد حالات الدراسة الدور المنميز

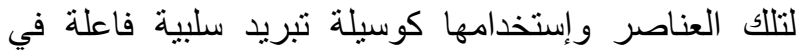

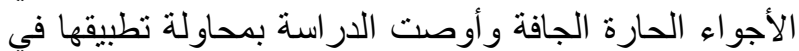

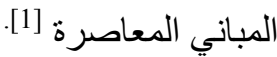

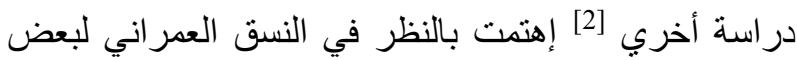

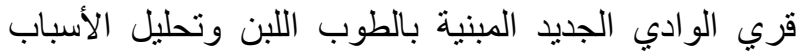

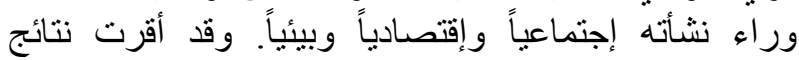

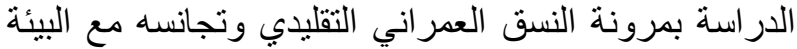
المحيطة وأن هذا النوع من التناسق بين البيئة المبنية النية النية

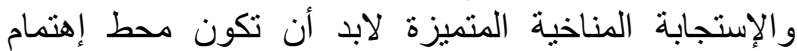

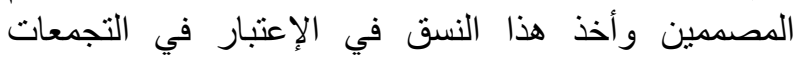
العمر انية المعاصرة. وفي دراسة مقارنه بين النسق العمر العاني

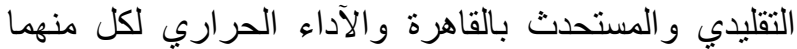

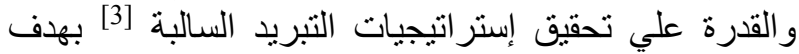

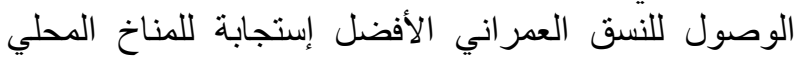
بالقاهرة. أكدت الدراسة علي أن النسق العدر العر اني التقليدي لله

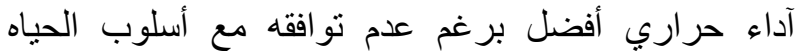

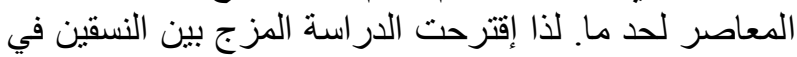
تكوين عمر اني يناسب الحياه المعاصرة من ناحية ويحقق آداء التراء حراري متميز من خلال إستخدام مفردات وإستراتيات التيجيات التجواب مع البيئة المستلهمة من النسق التقليدي. 


\section{Vol.41, No. 1. January 2022}

الدراسة معالجة كامله لهذا النموذج ونظام التهوية به محققة تحسين بنسبة (300 \%) ومع ذلك أوصي الباحث بإعادة

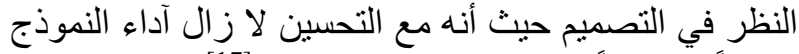

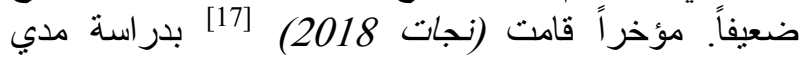

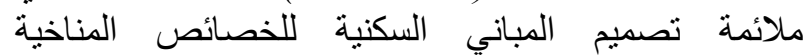

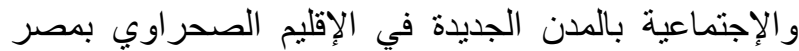
متخذة دراسة حالة نموذج الإسكان الإجتماعي المنفئة الإنف بمدينة

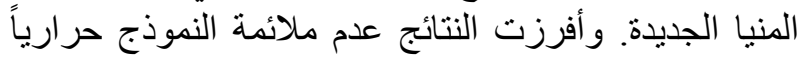

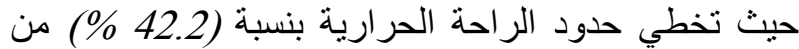

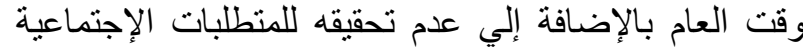

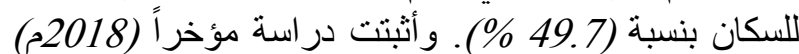

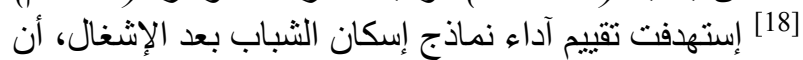
التعديلات التي أجريت بواسطة السكان علي تصميم الوحدة التئل

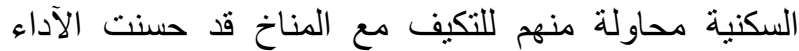
الحراري للمباني بشكل مؤثر وبنسبة نراوحت بين (60 \% .

وفي مجال الآداء الحراري لنماذج المدارس، قام (جادو

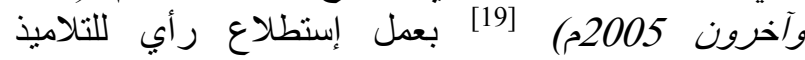
و المدرسين المستخدمين لثمانية عشر مدرسة بمحافظة المنيا

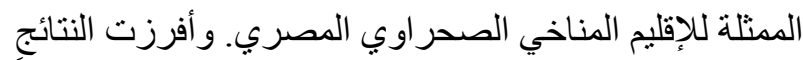

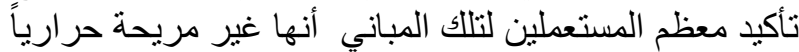

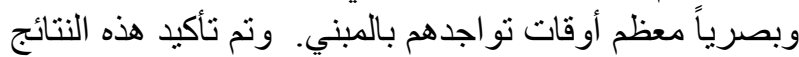

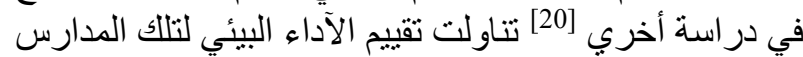

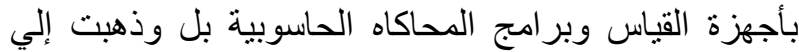

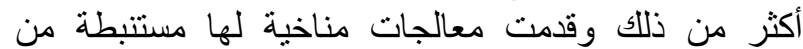

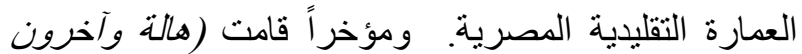

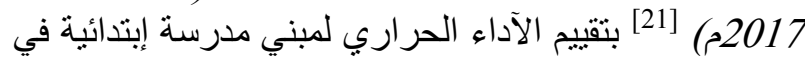

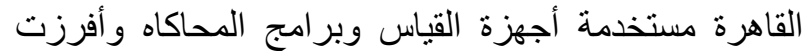

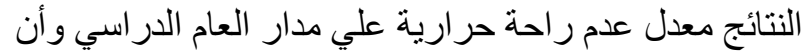

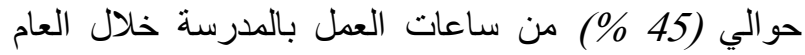

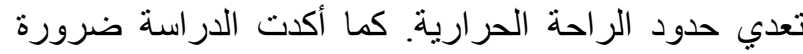

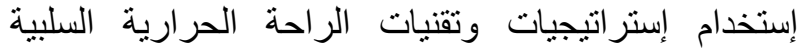

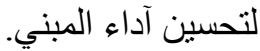

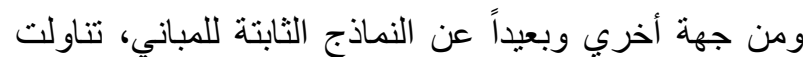

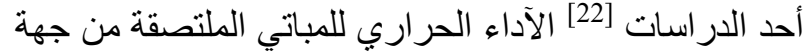

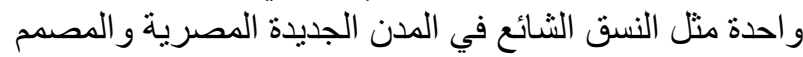
بمعرفة الأفراد وبحسب رالنبات فياتهم، وقد كانت نتائج المحاكاه التي أجرتها الدراسة لبست ببعيده عن الدراسات الدات سالفة الذكر حيث أكدت ضعف الآداء الحراري لهذه المبات الدباني مع تقديم

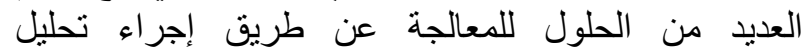

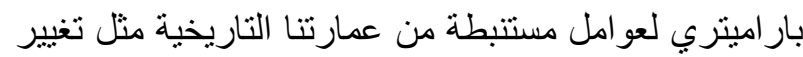

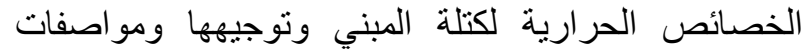
الفتحات وعناصر الإظلال علي كتلة المبني و التهوية الطبيعية والتي بتطبيقها أجرت تحسن ملموس ومؤثر في الإني الآداء Night (الحراري للمبني خاصة إستر اتيجية التهوية الليلية التئن [23 [بrge ventilation بدراسة الراحة الحرارية داخل ستة قاعات تعليمية بكلية
الراحة الحرارية داخل تلك المباني ومنها من قام بمحاولة

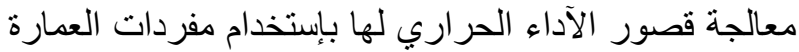

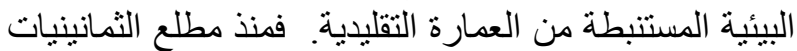

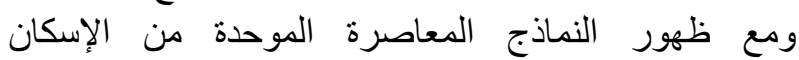

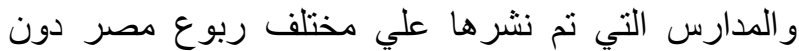
إعتبار لإختلاف الخصائص المناخية للأقاليم المصرية

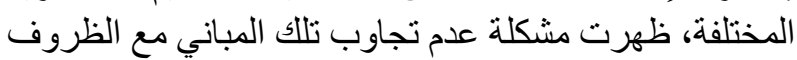

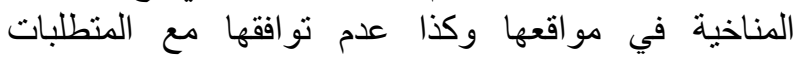

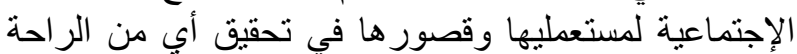

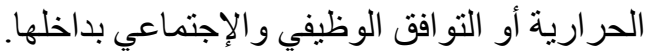

لاحظ (جون هاريس 1982) [12] أن النماذج السكنية

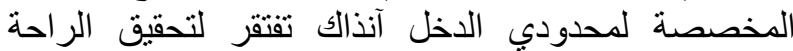

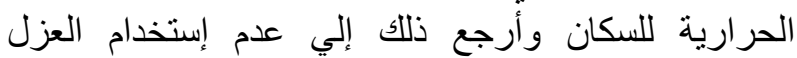

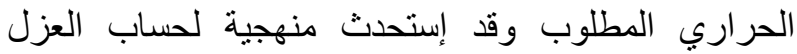

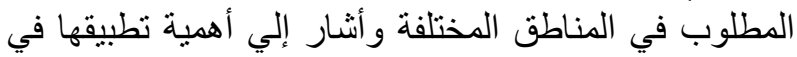

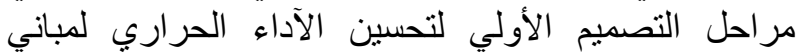

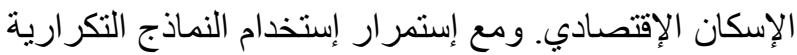

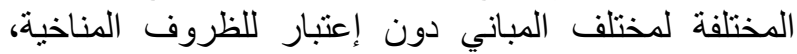

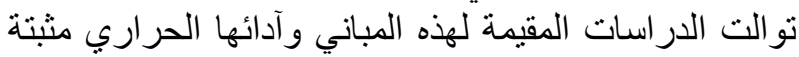

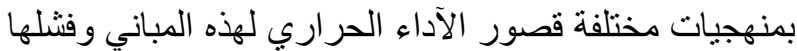

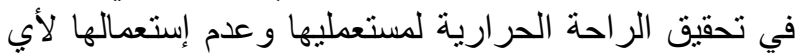

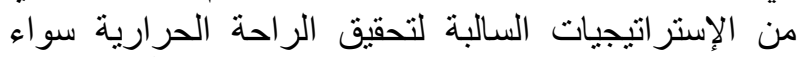

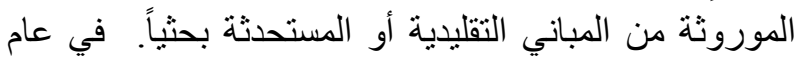

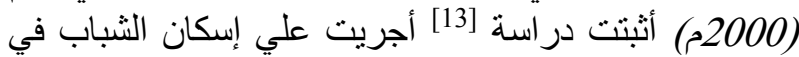

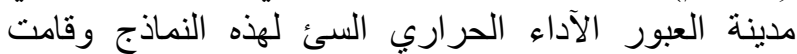

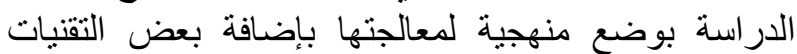

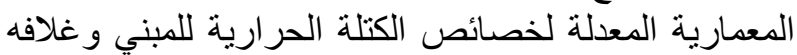
الخارجي وأوصت بتنفيذ تلك التقنيات حال إستخدام هذا لإنيا النموذج مرة أخري.

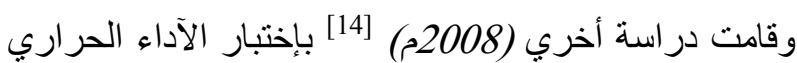

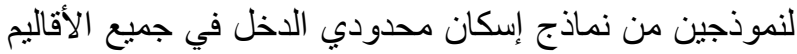

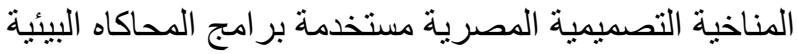

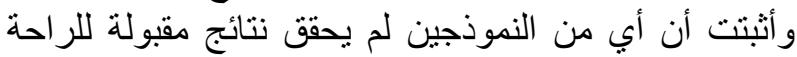

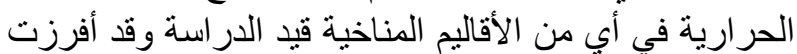

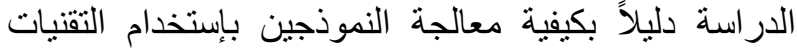

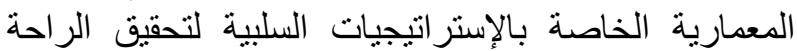

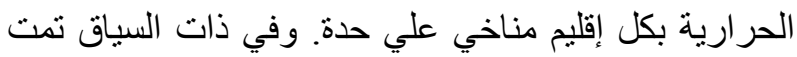
دراسة ثلاث نماذج إسكان لمحدودي الاخل بالمان بالمنيا كممثلة

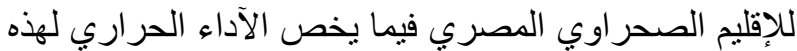

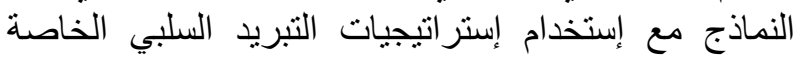

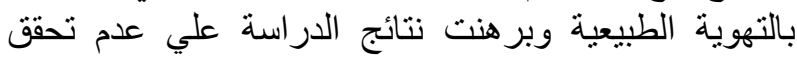

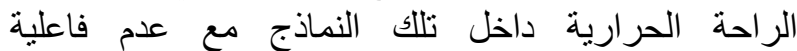

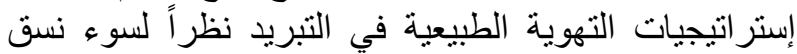

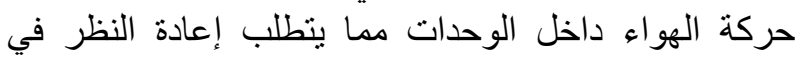

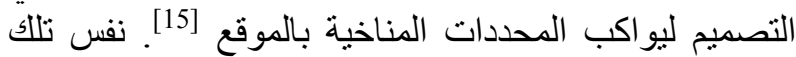

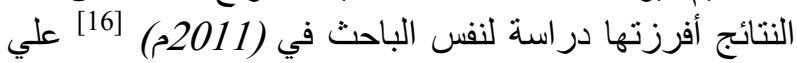
نموذج الإسكان القومي في نفس الإقليم المناخي وقدمت هذانه 
(region (Desert region) الصحر اوي شديد الجفاف (Dery dry desert region)

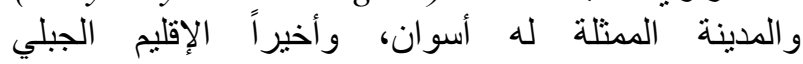
(Mountain region)

ويعتبر الإقليم الصحر اوي أكبر الأقاليم المناخية مساحة حيث

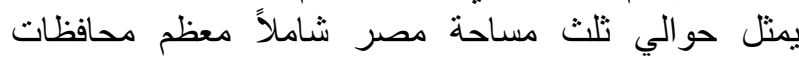
الصعيد مع غالبية مساحة الصحر اء الغرة الغربية (شكل رقم 1).

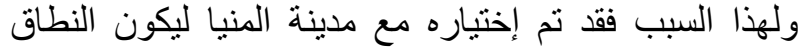

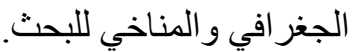
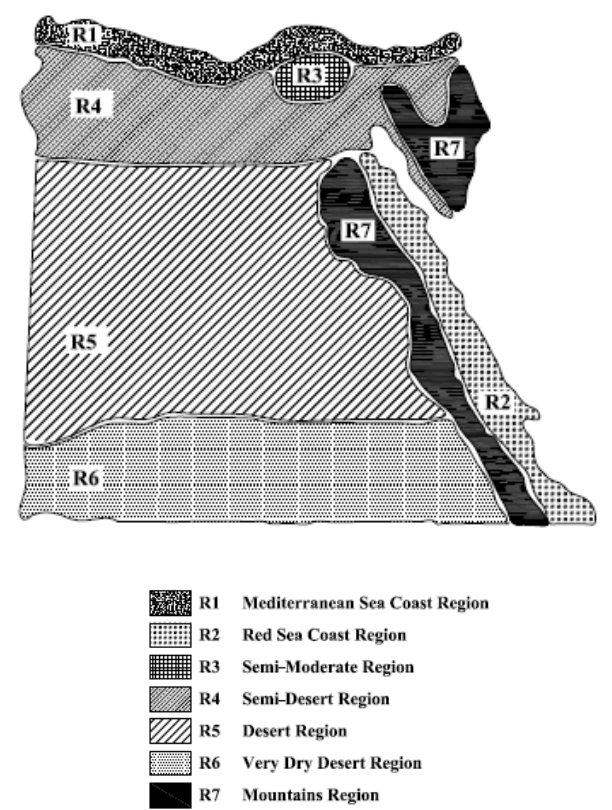

شكل رقم (1): الأقاليم المناخية التصميمية لمصر [25]

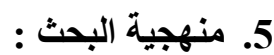

لتحقيق أهداف البحث والإجابة علي أسئلته البحثية إعتمد

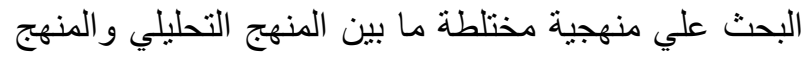
الإستقصائي (شكل رقم 2).

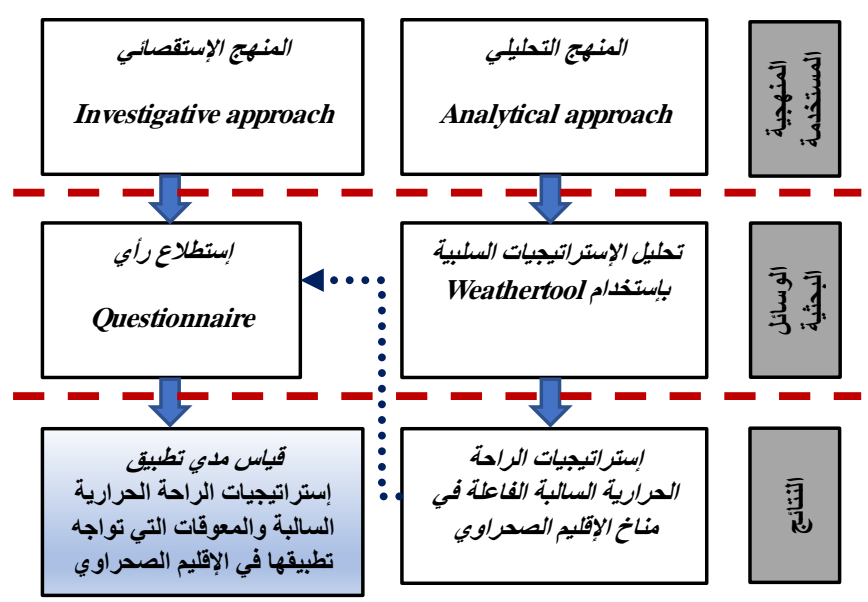

الهندسة - جامعة أسيوط وأقرت النتائج بأن القاعات غير مريحة حر ارياً في معظم الأوقات وهذا ما أكده (83 \% الطلاب مستخدمي تلك الفر اغات الدر اسية.

مما تقدم تظهر مشكله عدم تحقق الر احة الحرارية في المباني

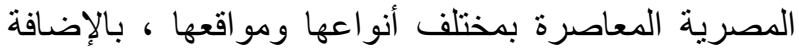

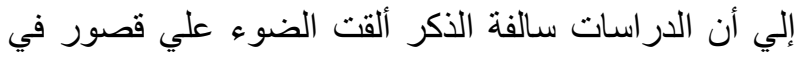

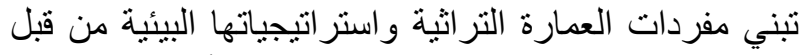

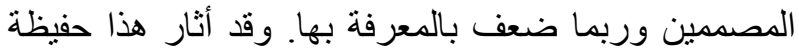

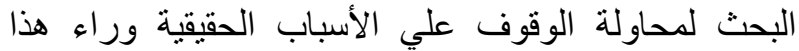

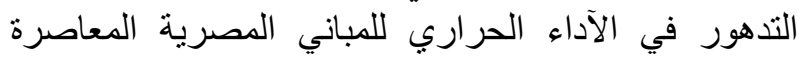
خاصة في الإقليم المناخي الصحر اوي المصري الصري.

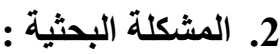

تتمثل إنكالية البحث في عدم وجود أسباب واضحة للقصور

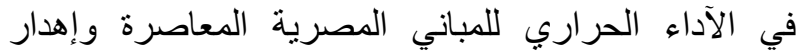

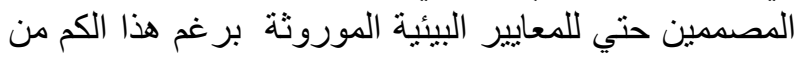

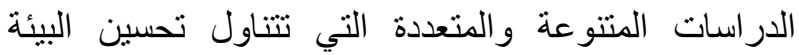

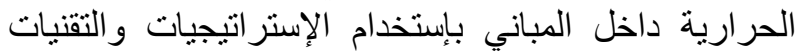
السلبية في أقاليم مصر المختلفة.

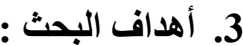

يهدف البحث إلي الوقوف علي الأسباب الحقيقية وراء هذا

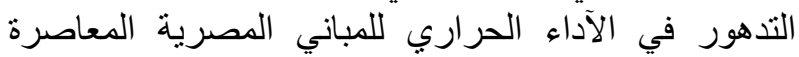

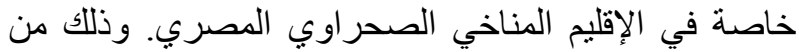
خلال محاولة الإجابة علي الأسئلة التالية:

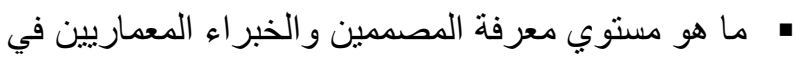
الإقليم بالإستراتيجيات السالبة الفاعلة لتحقيق الرئية الراحة

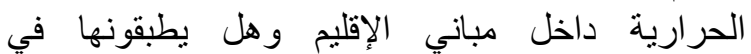
تصميماتهج? • ما هي المعوقات التي تعوق تطبيق تلك الإستر اتيجيات؟

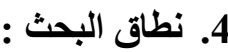

تتنوع الأقاليم المناخية التصميمية المصرية في خصائصها

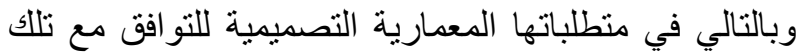

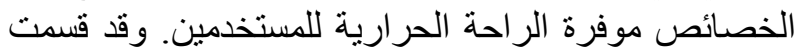

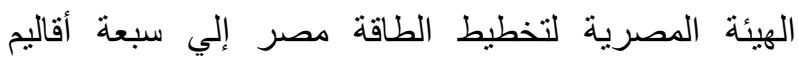
تصميمية مناخية هي] [20,24] : (شكل رقم 1) إقليم ساحل البحر

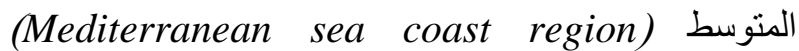
والمدينة الممثلة له الإسكندرية، إقليم ساحل البحر الأحمر (Red sea coast region) الإقليم شبه المعتدل (Semi-moderate region) و المدينة Semi-desert ( المثلة له طنطا، الإقليم شبه الصحر الهن 
Vol.41, No. 1. January 2022

السؤال السابق تحديد السبب بين مجموعة من الأسباب

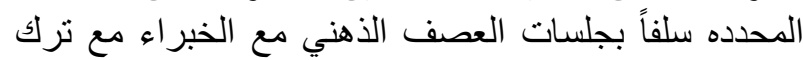

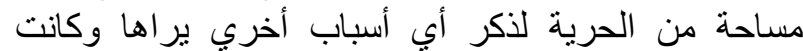

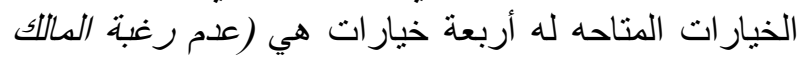

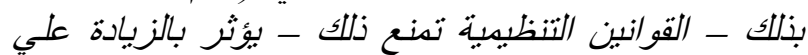

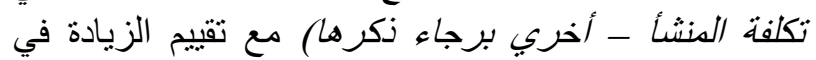

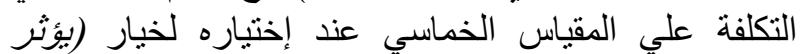
بالزيادة علي تنكلفة المنشأ).

نم تصميم إستمارة إستطلاع الر أي ، و لإز الة أي غموض بها

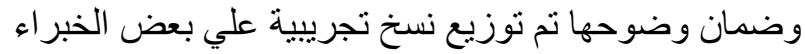

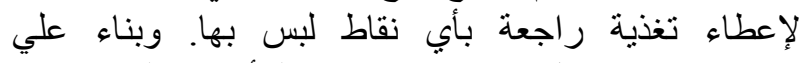
ملاحظاتهم تم تعديل إستمارة إستطلاع الر أي و عملها بصورة لئاء

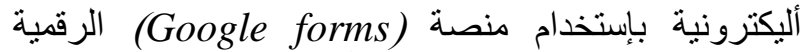

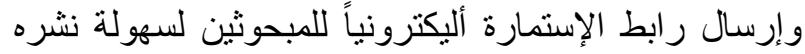

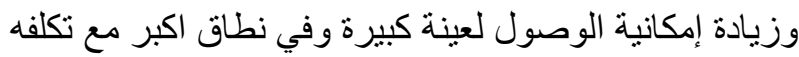

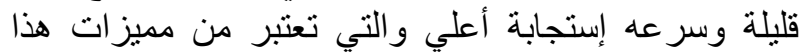
الإسلوب في جمع البيانات [29]

6. تحليل الإستر اتيجيات السالبة وتأثير ها علي الراحة الحرارية في مناخ المنيا :

الظروف المناخية القاسية في الإقليم الصحراوي المصري

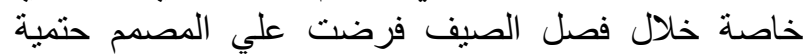

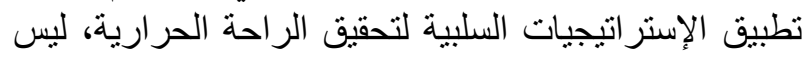

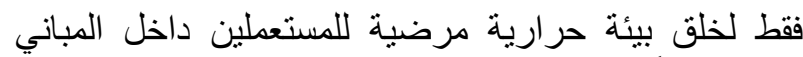

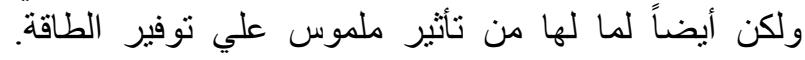

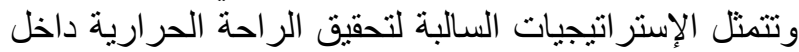

$$
\text { المباني في ست إستر اتيجيات رئيسية وهي: }
$$

• • • (heavy thermal mass

إستراتيجية التهوية الليلية مع كتلة حرارية معرضة Night purge ventilation + exposed (لها (thermal mass

Passive ) إستراتيجية التدفئة الثمسية السالبة (solar heating

Natural ) إستراتيجية التهوية الطبيعية (ventilation

Direct ) إستراتيجية التبريد بالتبخير المباثر (evaporativecooling (evaporative cooling • إستراتيجية التبريد بالتبخير غير المبر (Indirect evaporative cooling)

يختلف تأثثر هذه الإستراتيجيات من مكان لآخر بإختلاف

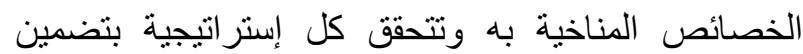

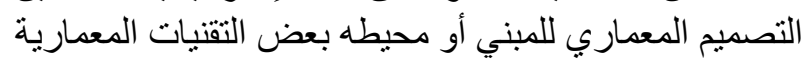

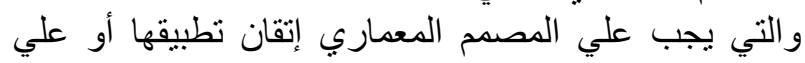

شكل رقم (2): المنهجية العامة للبحث و الوسائل البحثية و النتائج المستهدفة

\section{1 المنهج التحليلي للبحث :}

يههف الجزء التحليلي إلي تحديد أهم الإستر اتيجيات السالبة

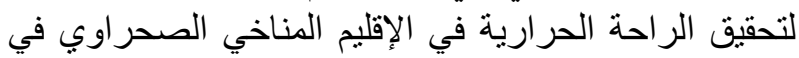

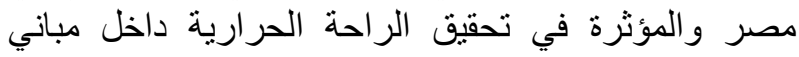

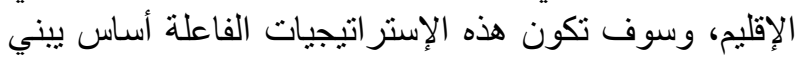

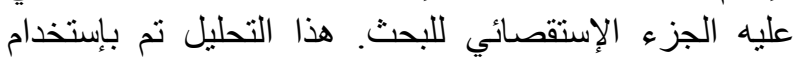

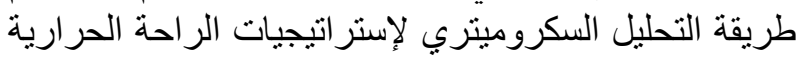

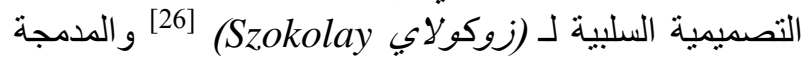
في برنامج (Autodesk Weathertool) الحاسوبي لتحليل

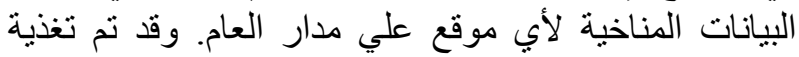

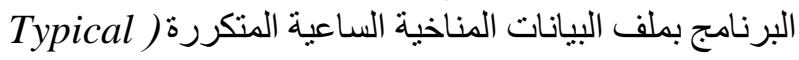
(Metrological Year - TMY file

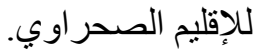

\section{5}

أما الجزء الإستقصائي في المنهجية فقد تبني وسيلة إسنطلاع

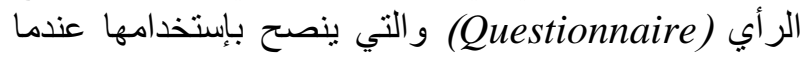
ينطلب الأمر الحصول علي معلومات بكم كبير لعينة كبيرة

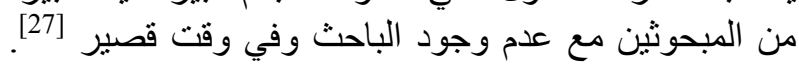

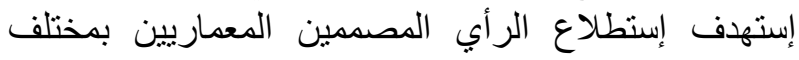

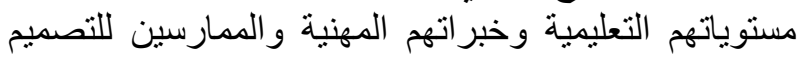

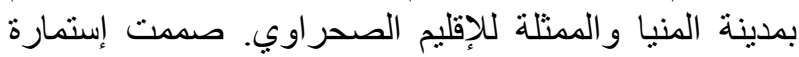

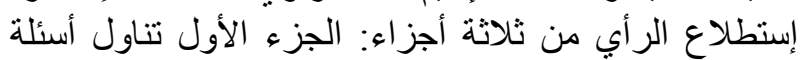

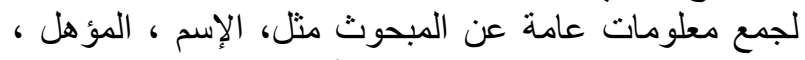
سنوات الخبرة في مجال التصميم. أما الجزء الثئ الثاني فقد تبني الإني

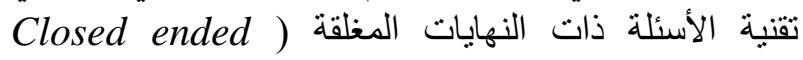
متعددة الخيار ات لسهولة التحليل الكمي للبيانات (questions

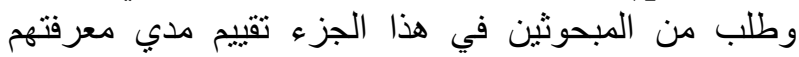

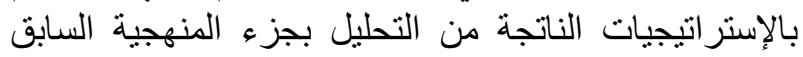
علي المقياس الخماسي لليكرت (Likert 5 points' scale)

وأخير اً فقد هدف الجزء الثالث و الأخير من إستطلاع الرأي

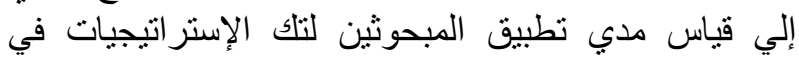

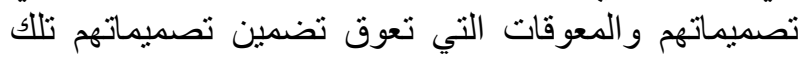

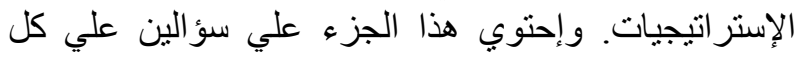

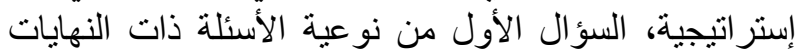

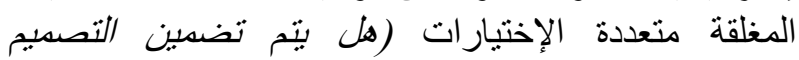

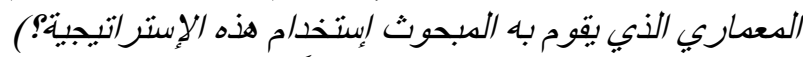

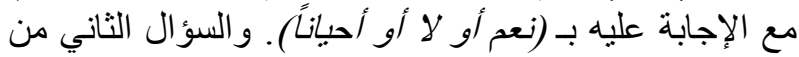

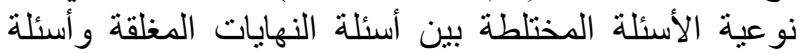

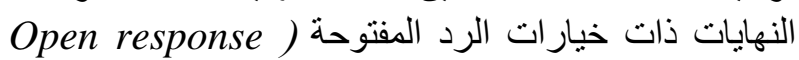
(option question 


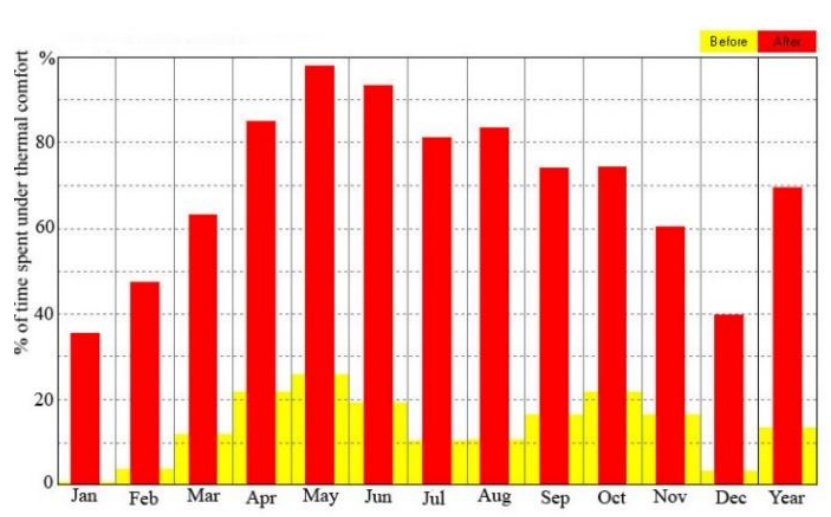

شكل رقم (4): تأثير إستر اتيجية الكنلة الحرارية المعرضة للتهوية الليلية علي الراحة الحرارية بالمنيا الباحث].

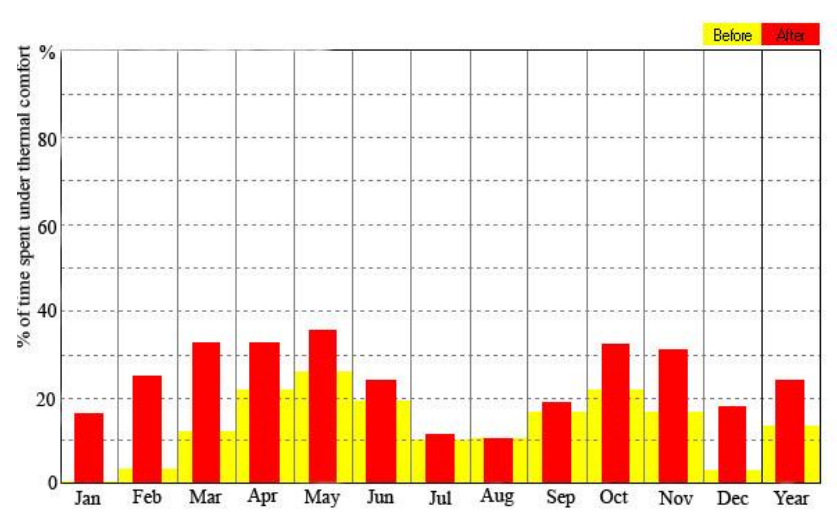

شكل رقم (5): تأثير إستر اتيجية التدفئة الثمسية السالبة علي الراحة

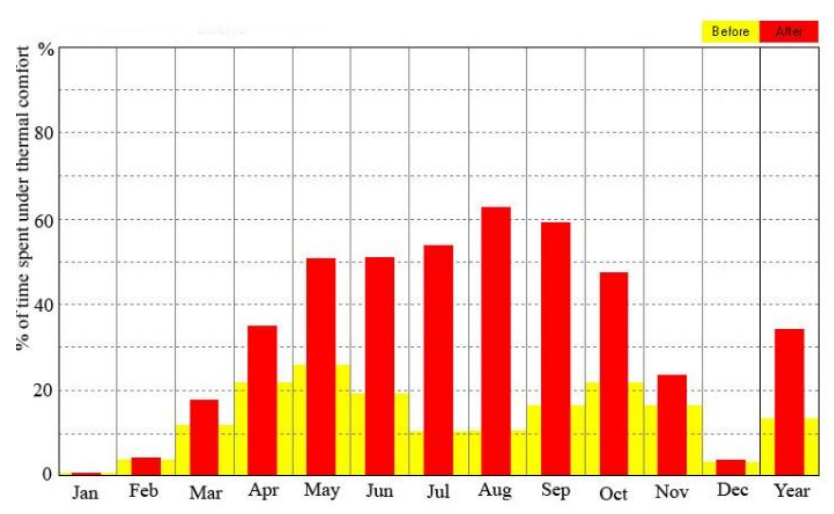

شكل رقم (6): تأثير إستر اتيجية التهوية الطبيعية علي الراحة الحرارية

الأقل إتقان تطبيق الإستراتيجيات المؤثرة بشكل كبير في إقليمه.

تبين الأشكال (3- 8) نتائج تحليل الإستراتيجيات السالبة

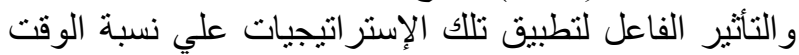
الذي يقضيه المستعملين للمباني بمدينة المنيا في منطقة الإنيات

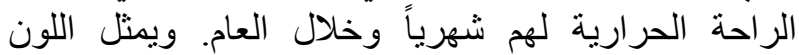

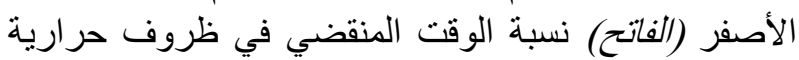

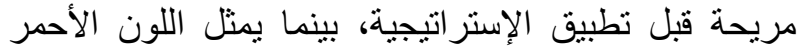

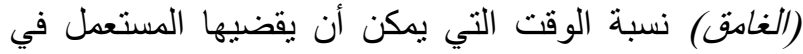

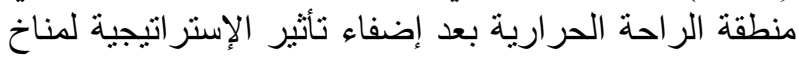
المنيا كممثل للإقليم الصحر اوي الصدة المصري قيد الدراسة.

كمتوسط سنوي جاء تأثثير إستر اتيجية إستعمال كتلة حرارية

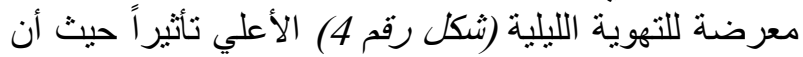

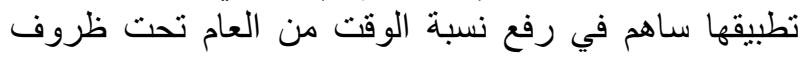

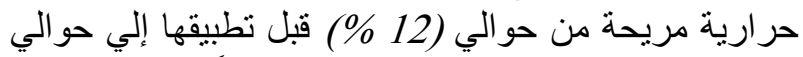

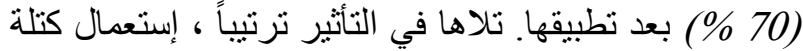

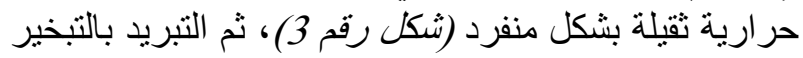

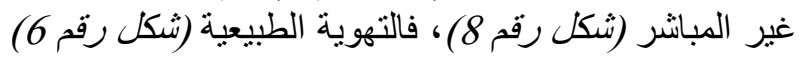

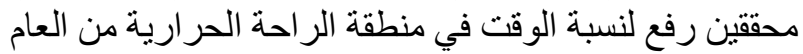

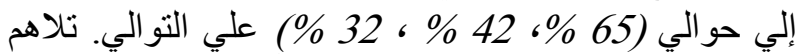
في المركز الأخير بنسب متساولة

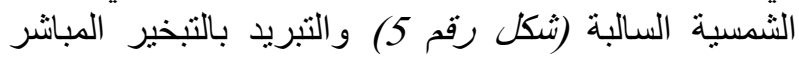
(شكل رقم 7) محققتين رفع لنسبة الوقت في منطقة الراحة الترة

$$
\text { الحر ارية من العام إلي حوالي (22 \% ع). }
$$

كذلك تظهر نتائج التحليل أن التأثير الأكبر كان لإستر اتيجيات

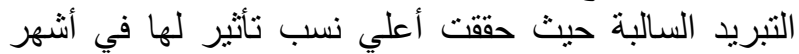

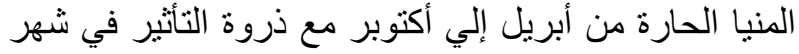

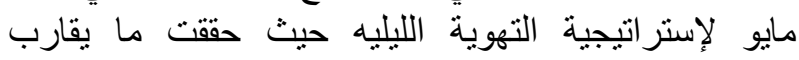
(98\%) من وقت الثهر تحت ظروف مريحة (شكل رقم 4). هذه النتائج تعطي مؤشراً و اضحاً أن مناخ المنيا كممثل فرئ

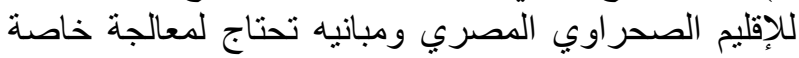

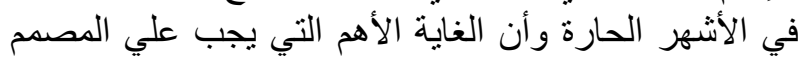
الحرص علي تطبيقها تتمثل في التبريد السلبي.

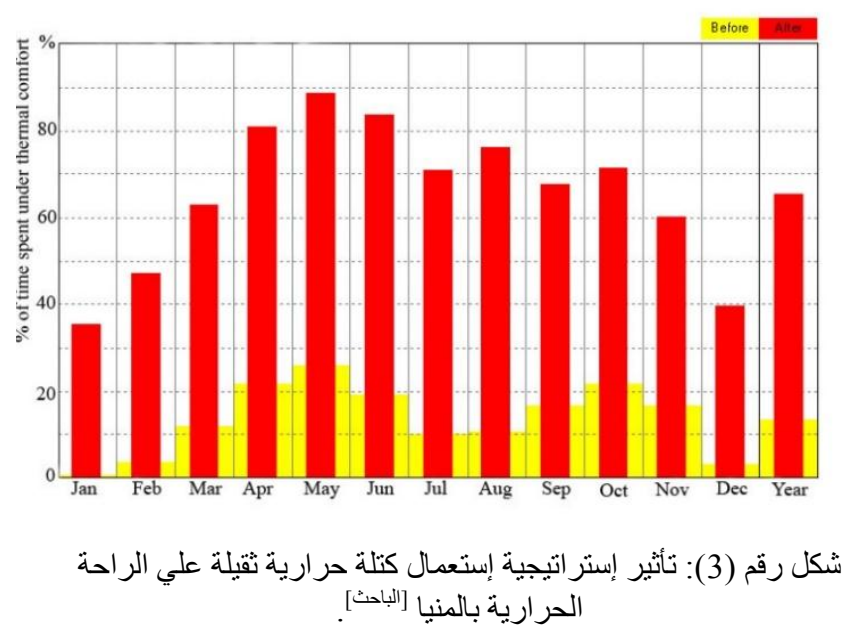


تم توزيع إستمارة إستطلاع الرأي أليكترونياً علي عدد كبير

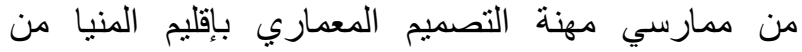
مستويات تعليمية وخبرات تطبيقية منباينة وفتحت الإستمارية

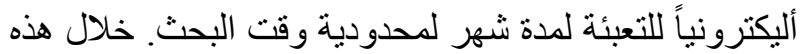

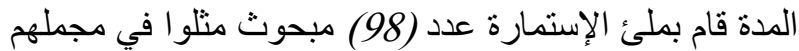
عينة الدراسة. تباينت المستويات التعليمية للمبحوثين (شكل

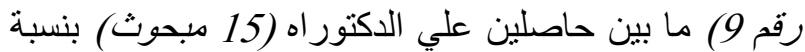

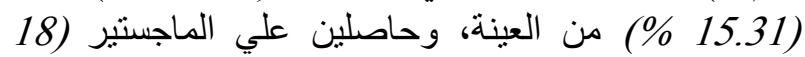
مبحوث) بنسبة (18.37 \% \%) من العينة وحاصلين علي البكالوريوس (65 مبحوث) بنسبة (66.33 \% \%) من العينة. كما تباينت وتدرجت سنو ات الخبرة بين المبحوثين (شكل رقم 10)، فمنلت الثريحة الكبري من المبحوثين من لديهم خبرة

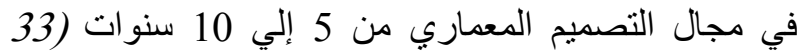
مبحوث) وبنسبة (33.67 \% \% من العينة. تلتها شريحة من لديهم خبرة أقل من 5 سنوات (25 من مبحوث) بنسبة (25.51) \% من العينة. ثم جائت بالترتيب شر ائح الخبرة من 10 : 15 من 15

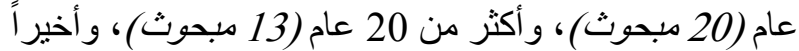
من 15 : 20 عام (7 مبحوثين) وبنسب من العينة تعادل (20.41 \%، 13.27 \%، 7.14 \% \%) علي الترتيب.

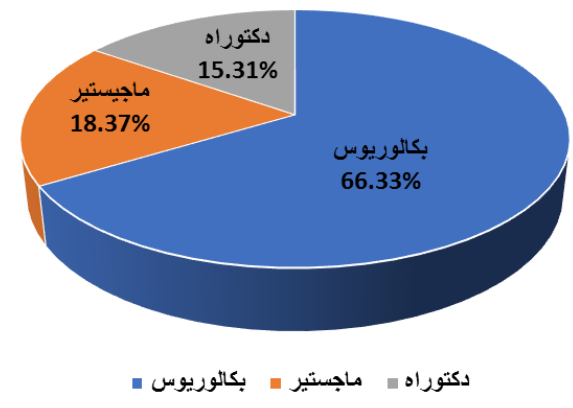

شكل رقم (9): نسب المستويات التعليمية المختلفة للمبحوثين [الباحث].

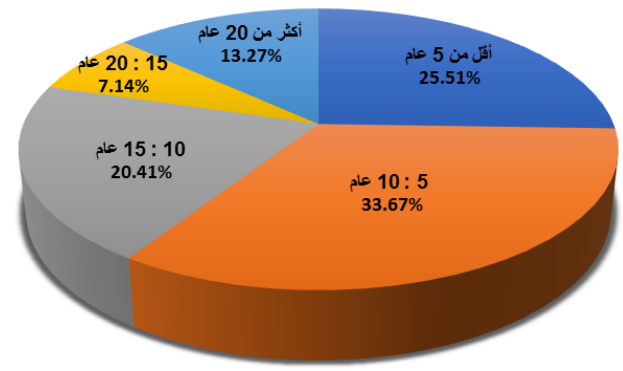

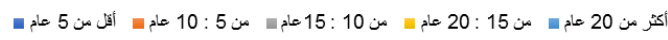

شكل رقم (10): نسب سنوات الخبرة المختلفة للمبحوثين [الباحث].

7 ـ 2 الجزء الثاني: قياس مدي معرفة المبحوثين بالإستراتيجيات قيد الاراسة ماني :

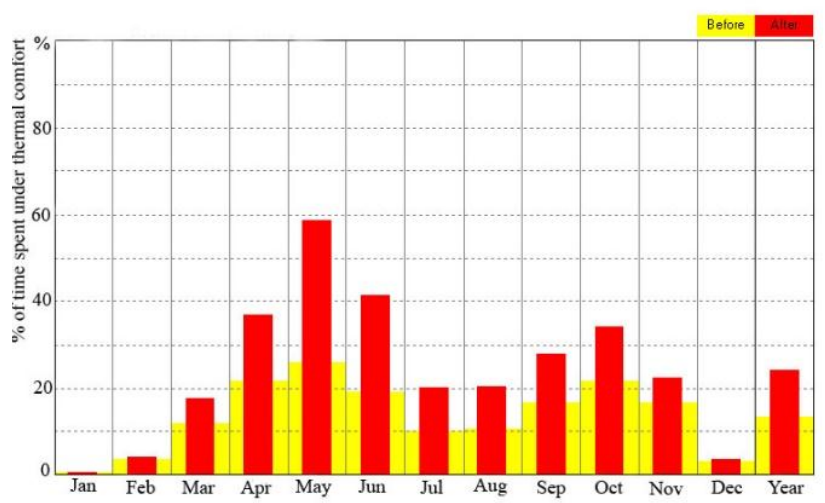
شكل رقم (7): تأثير إستراتيجية التبريد بالتبخير المباثر علي الراحة

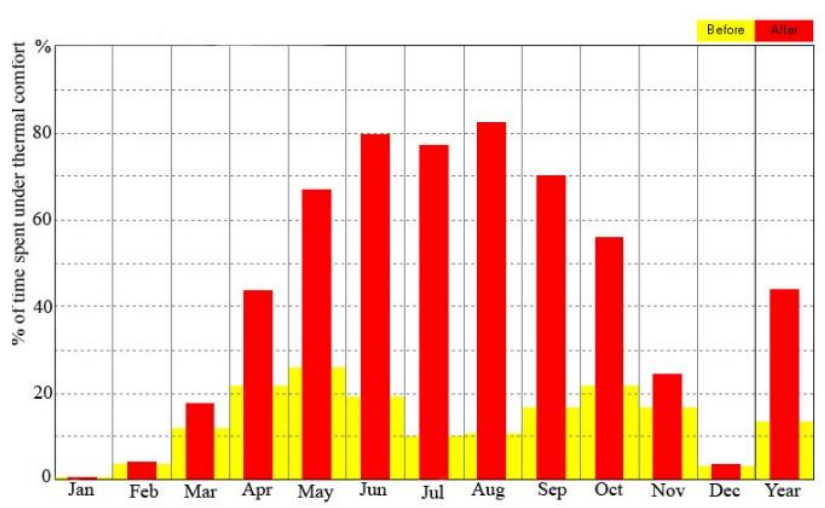

شكل رقم (8): تأثير إستر اتيجية التبريد بالتبخير غير المبانشر علي الراحة

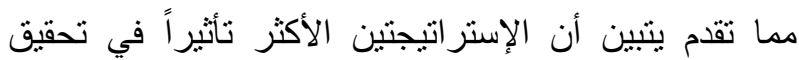

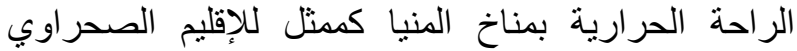

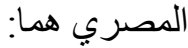

• إستر اتيجية التهوية الليلية مع كتلة حرارية معرضة لها Night purge ventilation + exposed) (thermal mass

Using heavy (إستر اتيجية إستعمال كتلة حر ارية ثقيلة (thermal mass

هاتان الإستراتيجيتان تمثلان الحد الأدني المطلوب معرفته

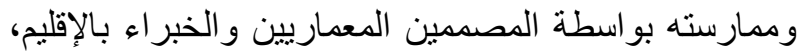

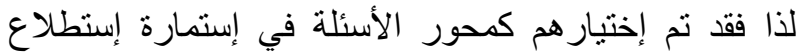

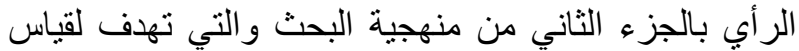

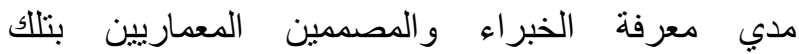
الإستر اتيجيات ومدي تطبيقهم لها و الوقوف علي أهم معوقات

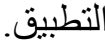

7. تحليل ومناقشة نتائج إستطلاع الرأي : 7 ـ 1 الجزء الأول: البيانات العامة للمبحوثين : 
Vol.41, No. 1. January 2022

ويوضح شكل رقم (12) الوزن النسبي لمعرفة المبحوثين

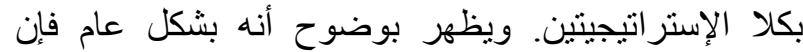

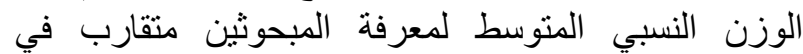
الإستر اتيجيتين حيث حققت إستر اتيجية إستعمال كتلة حرارية

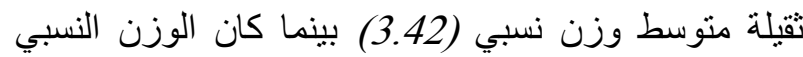
المتوسط(3.33) لمعرفتهم بإستر اتيجية التهوية الليلية.

وتعبر هذه النتائج عن معرفة أعلي قليلاً من المتوسطة

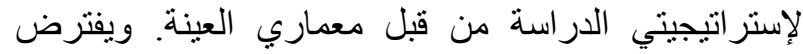
الباحث أن يكون هذا لضعف ما في مقررات الإندي التصميم البيئي

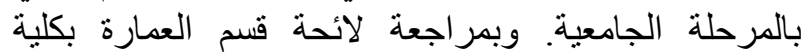

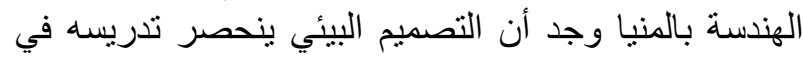

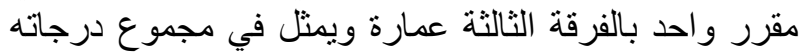
نصف مقرر عادي، مما يعضض ويثبت هذه الفرضية.

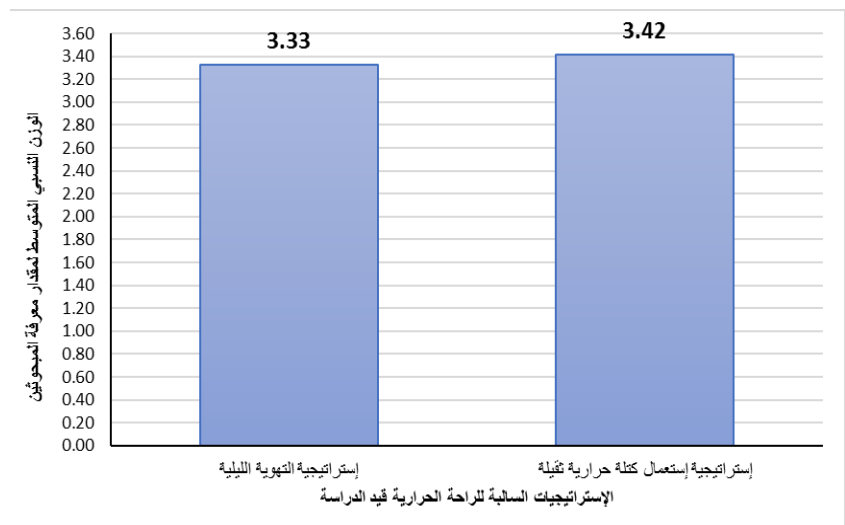

شكل رقم (12): الوزن النسبي المتوسط لمقدار معرفة المبحوثين

7 ـ 3 الجزء الثالث: قياس مدي تطبيق المبحوثين للإستراتيجيات قيد الدارسة ملئة ومعوقات التطبيق :

بينت أجوبة المعماريين المبحوثين عن ما إذا كانوا يقومون

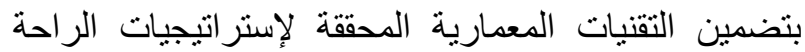

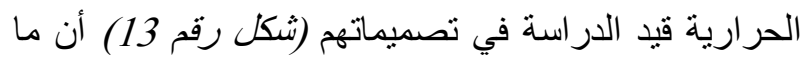

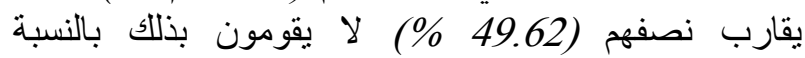

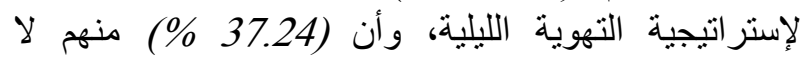
يستخدمون تقنيات إستراتيجية الكتلة الحرارية الثقيله في التية

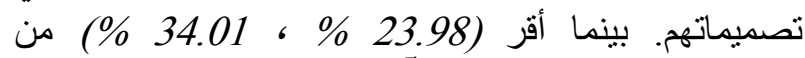

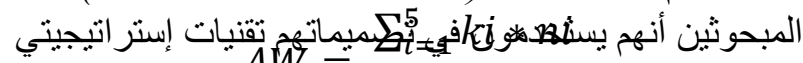

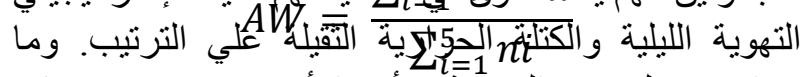
يتراوح حول ربع المبحوثين أقروا أنهم يستخدمون تقنيات الإستر اتيجيتين أحيانا وليس دائماً.
تحليل التقييم الذاتي للمبحوثين لمدي معرفتهم بتفاصيل الإستراتيجيتين قيد الدراسة (التهوية الليلية وإستعمال كتلة

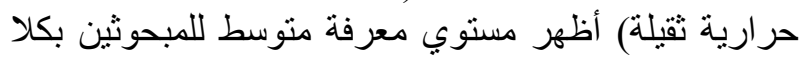

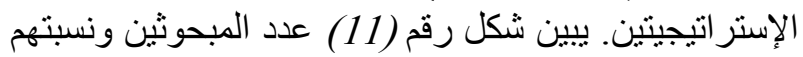

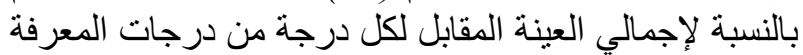

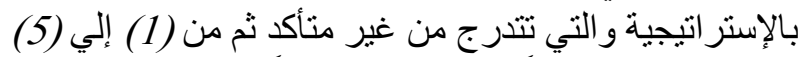
حيث (1) الأقل تقييماً و(5) الأعلي تقنيماً.

من خلال دراسة شكل رقم (11) يتبين أن النسبة الأكبر من المبحوثين (62.24 \%) أظهر معرفة تتراو ح ما بين المتوسط الاكير

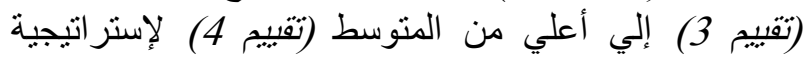

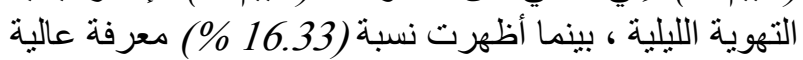
بنفس الإستراتيجية. أما بالنسبة لإسنراتيجية إستعمال كتلة الإنة حر ارية ثقيلة، فقد قيم السو اد الأعظم من المبحوثين (76.53) \%) معرفتهم بها من متوسطة (تقييم 3) إلي عالية (تقبيم 5) (5).

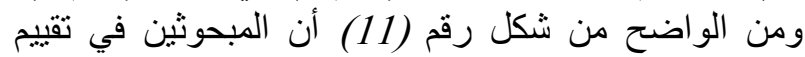

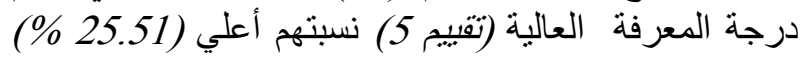

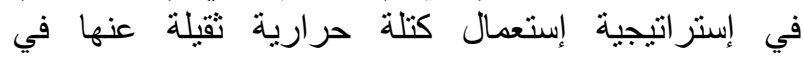
إستر اتيجية التهوية الليلية (16.33 \% \%).

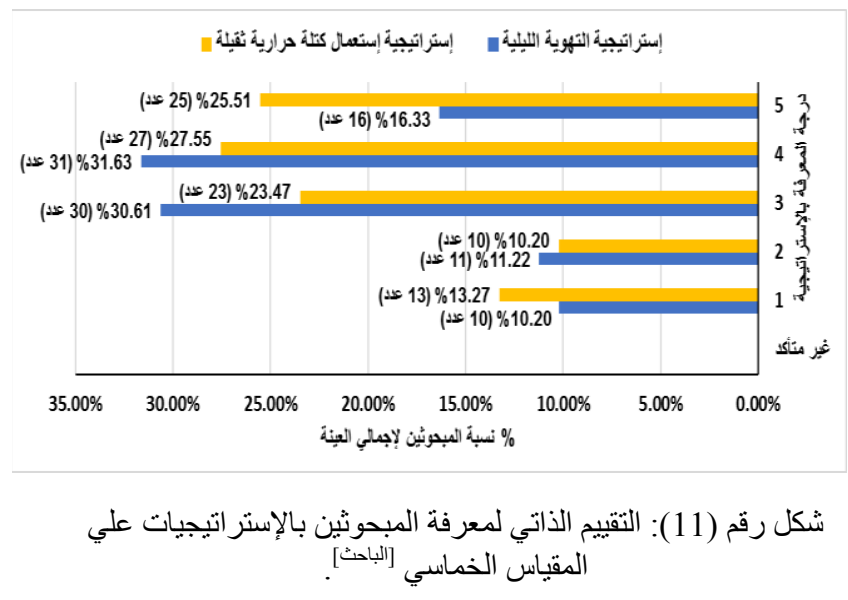

ولتقييم الوزن النسبي لمتوسط معرفة المبحوثين بإستر اتيجيتي الدر اسة تم إستعمال المعادلة التالية:

حيث:

= الوزن النسبي المتوسط للمعرفة بالإستر/تيجية =ki

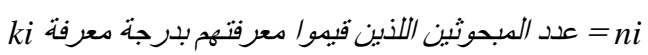


Vol.41, No. 1. January 2022

وأقرت نتائج تقييمه للزيادة في التكلفة بدرجة أقل من المتوسطة وبقيمة مؤشر تأثير علي التكلفة تعادل (2.64) لإستراتيجية التهوية الليلية أعلي منه في إستراتيلة التيجية الكتلة

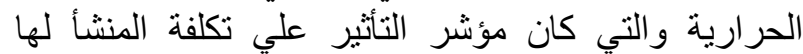

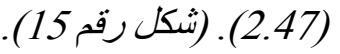

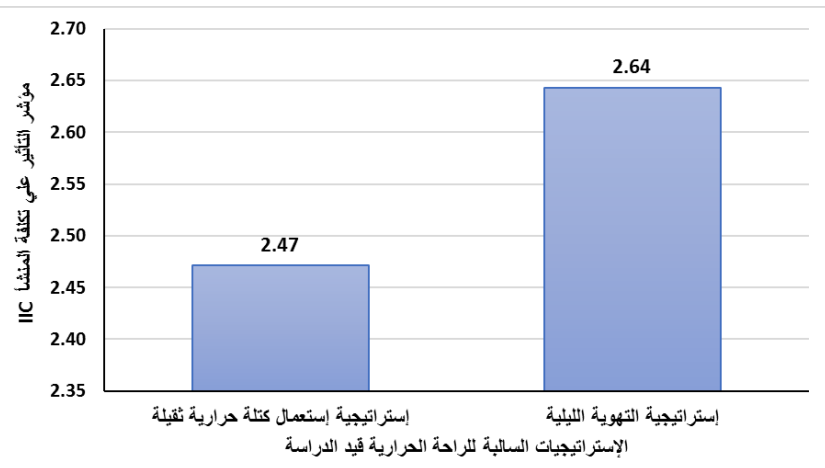

شكل رقم (15): مدي تأثير تطبيق الإستر اتيجيات قيد الدراسة علي زيادة

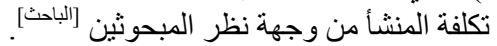

علاوة علي ذلك فإن العديد من المعوقات الأخري تم ذكرها بو اسطة المبحوثين و التي تعرقل تطبيق إستراتيجيات الرئ الراحة الحر ارية الفاعلة بالإقليم ويمكن تلخيصها في في الآتي:

بعض الإستراتيجيات تسبب هدر في المساحات وبذلك تزيد التكلفة ويقل العائد الإستثماري بعكس رغبة الملاك و المستثمرين.

عدم وجود الوعي والمعرفة الكافية لائي الملاك و المصممين و عدم إقتناعهم بأهمية هذه الإسنر اتيجية الإية

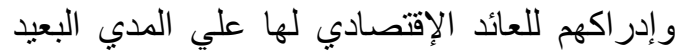

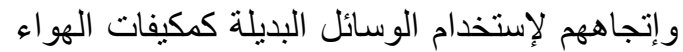

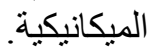

عدم تو افر العمالة الماهرة لتنفيذ التقنيات المعمارية لتلك الإستر اتيجيات مع القصور في صيانتها.

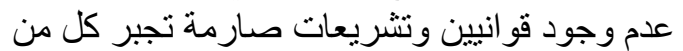

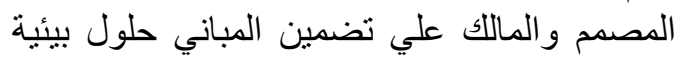
سليمة ومناسبة لمحيطه المناخي. القصور في التعليم المعماري الموجه للعمارة البيئية

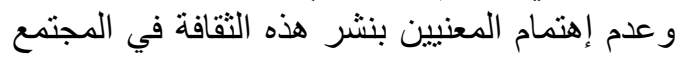

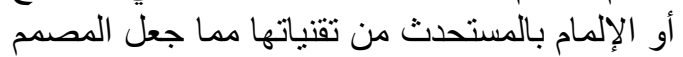
يهتم بالثكل دون الوظيفة ور احة المستعطلين.

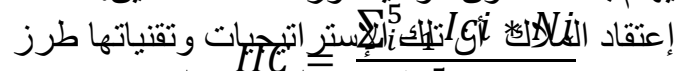
قديمة وتضطليه و إتجاههم لإستخدام بدائل تعطي للعقار ات مظهر

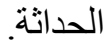
نو عبة المبني وظروف الموقع ومتطلبات المالك في

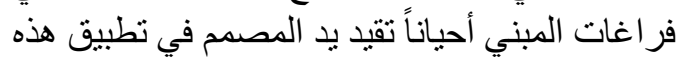

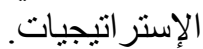

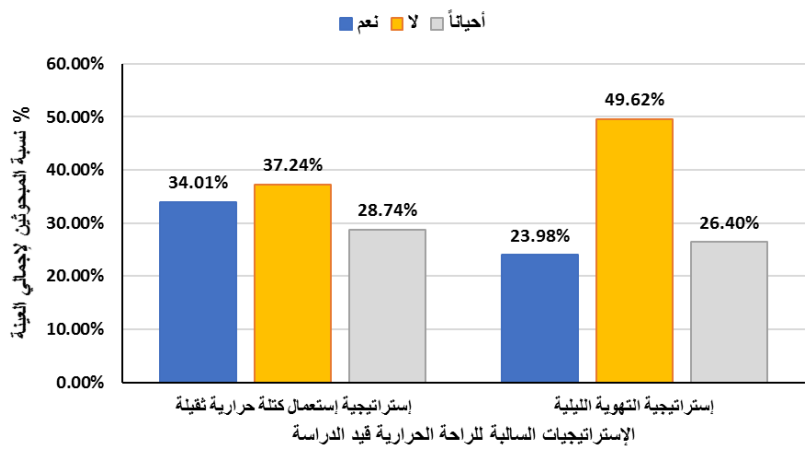

شكل رقم (13): مدي تطبيق المبحوثين للإستر اتيجيات قيد الدراسة في تصميماتهم المعمارية [الباحث].

وبسؤ الهم عن أسباب عدم إستخدامهم لهذه الإستر اتيجيات، أقر

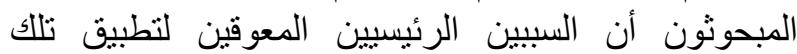

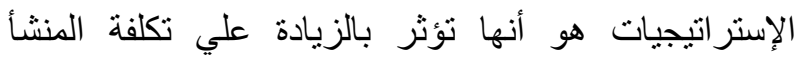

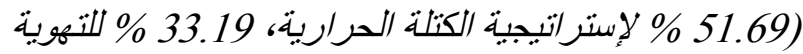

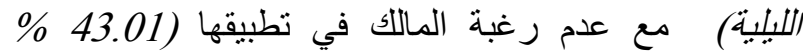

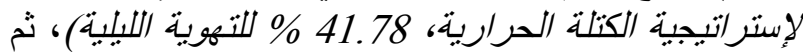

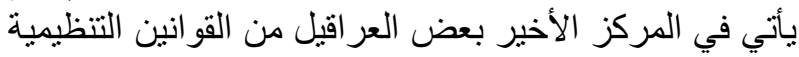

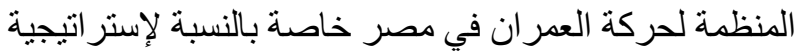
التهوية الليلية (شكل رقم 14).

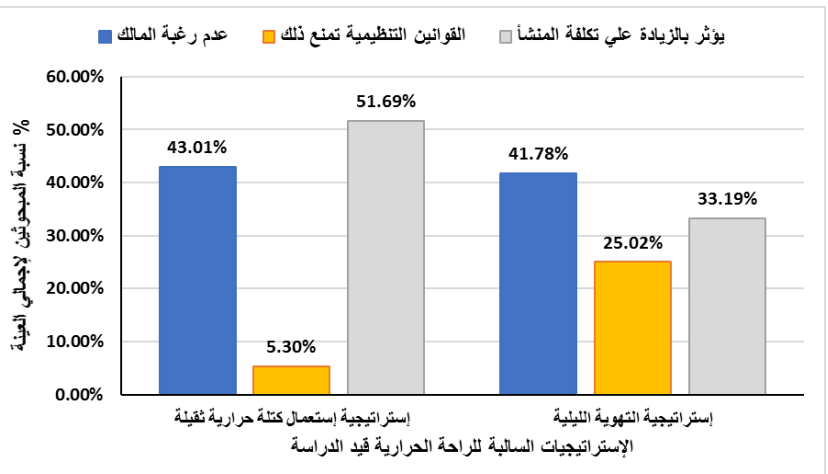

$$
\text { شكل رقم (14): معوقات مدي تطبيق الإستر اتيجيات قيد الدر اسة من }
$$

وقد قدر المبحوثون مقدار الزيادة في تكلفة المنشأ التي

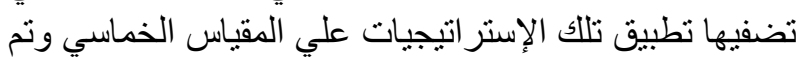

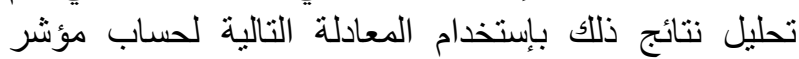
التأثير علي تكلفة المبني ( Impact Index for Cost : ${ }^{30]}$ (IIC

حيث:

(Impact Index for Cost) مؤشر التأثي علي تكلفة العبني =IIC Impact weight for cost) الوزن النسبي لتأثير التنكلفي Ici

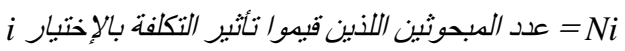


Vol.41, No. 1. January 2022

\% منهم لا يستعملون إستراتيجية الكتلة الحرارية

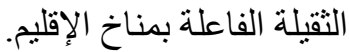

\section{8 ـ 3 المعوقات وراء ضعف تطبيق الإستراتيجيات السالبة بالإقليم الصحراوي المصري :}

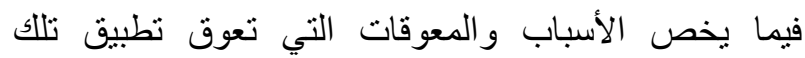

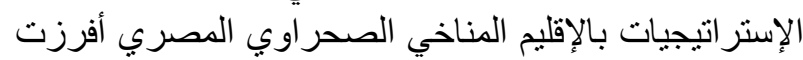

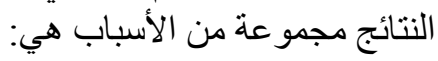

1- إضفاء تطبيق تلك الإستر اتيجيات زيادة علي التكلفة للمنشأ سواء زيادة مباشرة أو غبر مباثرة زبرة بتقليل العائد الإستثماري منها بهدر المادة المساحات و الحاجة

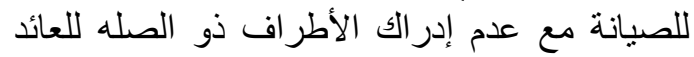
الإقتصادي لها علي المدي البعيد. وقدر هذا التئ التأثير التير بدرجة أقل من المتوسطة بمؤشر تأثثر علي التئلفي 2.64 IIC الحرارية الثقبلة).

2- عدم رغبة الملاك و المستثرين في تطبيقها

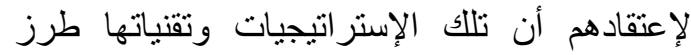
قديمة وتضفي مظهر تقلبدي علي عقار اتهم و إتجاههم

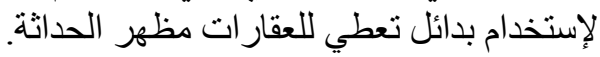
3- عراقيل التطبيق التي تضفيها القوانين المنظمة للعمران في مصر خاصة في تنفيذ تقنيات التهوية التئن

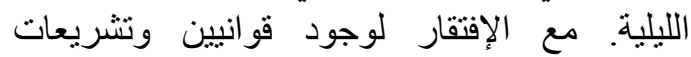
صارمة تجبر كل من المصمم و المالك علي تضمين المباني حلول بيئية سليمة.

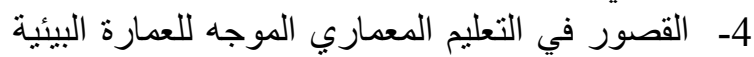

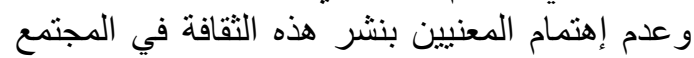

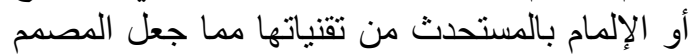
يهتم بالثكل دون الوظيفة ور احة المستعملين.

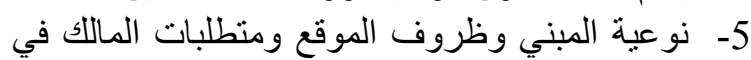

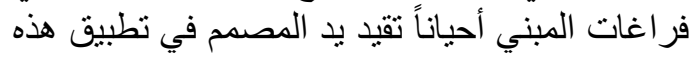

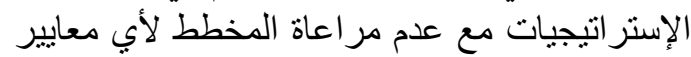
بيئية علي مسنوي المدينة تييسر مهمة المعماري في لاعي الئي التطبيق.

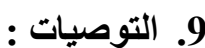
بناء علي ما تقلم يوصي البحث بالآتي:

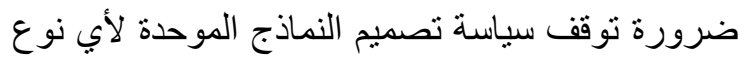

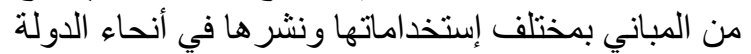
دون الأخد في الإعتبار تباين الظروف المنئن المناخية

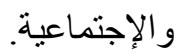
ضرورة حث المعمارين علي تبني تطبيق الإستر اتيجيات

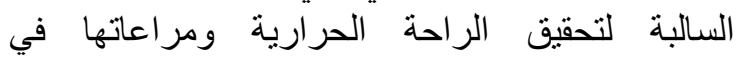

لا ير اعي التخطيط في مصر إلا الوظيفة للشوارع و الفر اغات مع إسقاط و اضح لأي معايير بيئية علي الائي

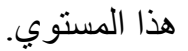

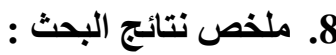

خلص البحث من خلال منهجيته و الوسائل البحثية التي إتبعها

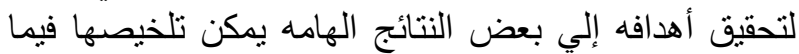

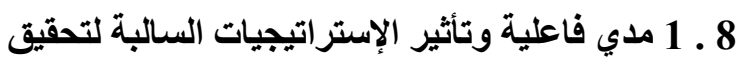
الراحة الحرارية في الإقليم الصحراويري الصئي المصري :

أظهرت نتائج تحليل الإستر اتيجيات السالبة في مناخ المنيا كممثل للإقليم الصحر اوي المصري تصني ما يلي:

1- فاعلية دور جميع الإستر اتيجيات السلبية في تحسين مستوي الراحة الحرارية عند تطبيقها علي الإني المباني

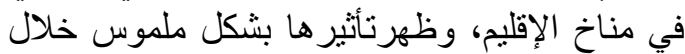

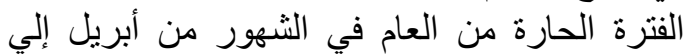

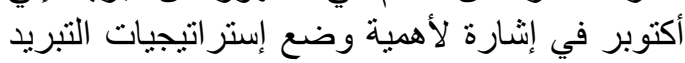
السلبي في الحسبان عند التصميم في هذا لإلقإليح.

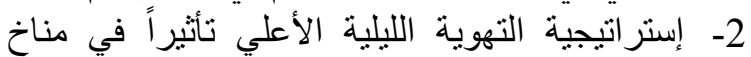

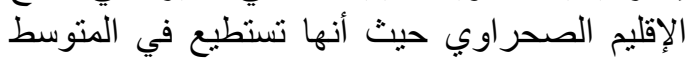

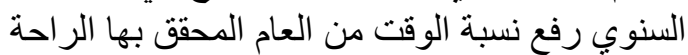

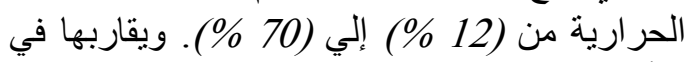

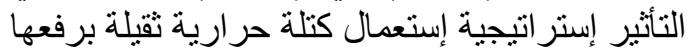

$$
\text { إلي (65 \% \%). }
$$

\section{8 السالبة ومدي تطبيقهم لها :}

فيما يخص مستوي معرفة المصمين و الخبراء المعماريين

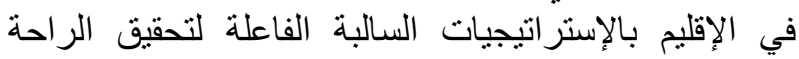

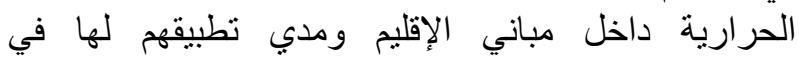
تصميماتهم، دللت النتائج علي:

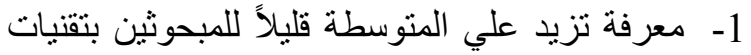

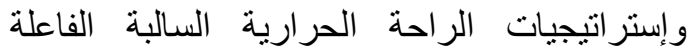
بالإقليم بمتوسط وزن نسبي (3.42 للكتلة الحرارية الكية

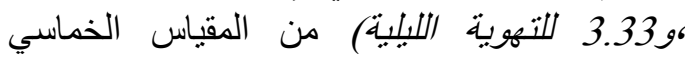
2 القصتور الثنديد في تطبيق الإستر اتيجيات السالبة في تصميم المباني بالإقليم حيث أن (49.62 \% المعماريين المبحوثين لا يطبقون أي من تقانيات المنيات إستر اتيجية التهوية الليلية في أعمالهم، وأن (37.24 الهنين 
Vol.41, No. 1. January 2022

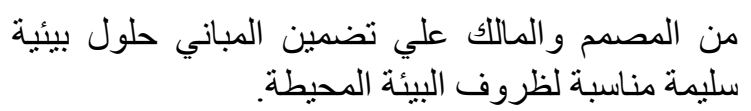

adaptively re -used historical palaces in Cairo, Egypt. 2001, The University of Wisconsin - Milwaukee: Ann Arbor. p. 287.

.11 Mohamed, M., Traditional Ways of Dealing with Climate in Egypt. 2010.

.12 Harris-Bass, J., Thermal Insulation of Low-Cost Housing in Egypt. Journal of Building Physics, 1982. 5.(153)

.13 El-Hefnawi, A.I.K., Climatic design for lowcost housing in Egypt "Case of the Youth Housing Project in El-Obour City", in AEE "Architecture, Energy \& Environment" Tools for climatic design - Advanced International Training Programme. 2000, Lund University: Lund Sweden.

.14 Moustafa, W.S.A., The climatic design of residential buildings in Egypt: Case study Mansoura city, in Department of Architecture. 2008, Mansoura University: Mansoura, Egypt.

.15 Gado, T. and M. Osman, Investigating natural ventilation inside walk-up housing blocks in the Egyptian desert climatic design region. International journal of ventilation, 2009. 8(2): p. 145-160.

.16 Osman, M.M.A., Evaluating and enhancing design for natural ventilation in walk-up public housing blocks in the Egyptian desert climatic design region, in Dundee school of architecture. 2011, University of Dundee.

.17 Makhlouf, N.A., The suitability of residential buildings design to climatic and social attributes within the new cities of the Egyptian desert region (With application to New Minya city), in Architectural Engineering Dept. 2018, Minia University.

.18 Moustafa, W.S., M.M. Abdelrahman, and I.R. Hegazy, Building performance assessment of user behaviour as a post occupancy evaluation indicator: Case study on youth housing in Egypt. Building Simulation, 2018. 11(2): p. 389-403.

.19 Gado, T., M. Mohamed, and S. Unwin. The environmental performance of classrooms in Egypt: a case study from ElMinya governorate. in The Second Scottish Conference for Postgraduate Researchers of the Built \& Natural Environment (PRoBE 2005). 2005. Glasgow Glasgow Caledonian University.

.20 Mohamed, M., Investigating the environmental performance of government primary schools in Egypt: With particular concern to thermal comfort, in Dundee

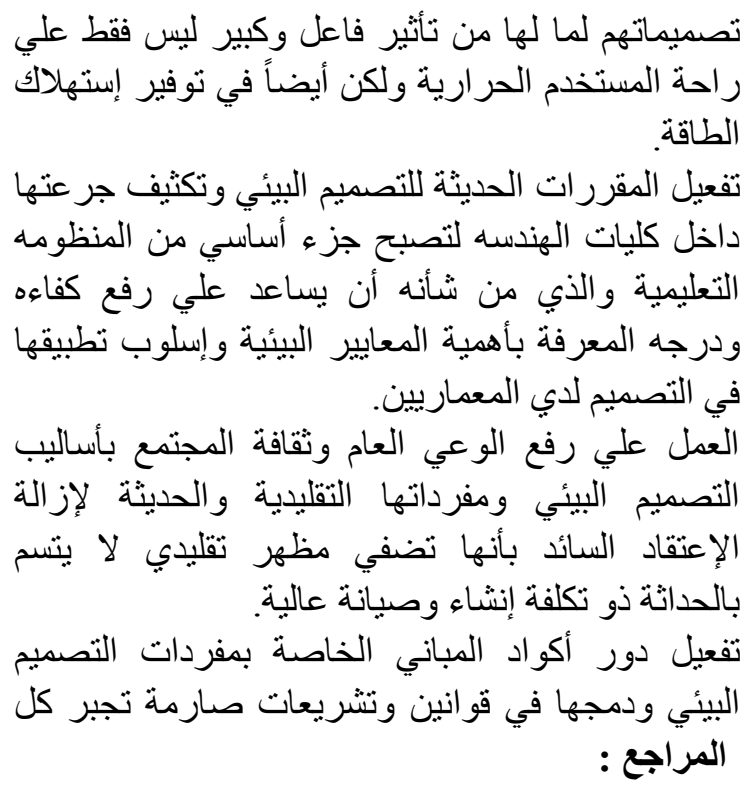

.1 Attia, S. The role of landscape design in improving the microclimate in traditional courtyard-buildings in hot arid climate. in PLEA2006 - The 23th conference on passive and low energy architecture. 2006. Geneva - Switzerland: Universite de Geneve.

.2 Balbo, R., Shape, culture and environment: a lesson of urban design from Dakhleh oasis, Egypt, in PLEA2006 The 23rd Conference on Passive and Low Energy Architecture. 2006: Geneva, Switzerland.

.3 Fahmy, M. and S. Sharples, Passive design for urban thermal comfort: A comparison between different urban forms in Cairo, Egypt, in PLEA 2008 - 25th Conference on Passive and Low Energy Architecture, P. Kenny, V. Brophy, and J.O. Lewis, Editors. 2008, University College Dublin: Dublin, Ireland.

.4 Sameh, H., A.E. Zafrany, and D.N. Attiya, Analysis of thermal comfort enhancement using vernacular architecture in Siwa Oasis, Egypt. Journal of Engineering and Applied Science, 2019. 66(6): p. 679-701.

.5 Fathy, H., Natural energy and vernacular architecture principles and examples with reference to hot arid climates. 1986, Chicago and London: The university of Chicago press

.6 Fernandes, J., et al. Thermal performance and comfort of vernacular earthen buildings in Egypt and Portugal. in International Conference on Vernacular Earthen Architecture, Conservation and Sustainability, SOStierra 2017, September 14, 2017 - September 16, 2017. 2018. Valencia, Spain: CRC Press/Balkema.

.7 Gado, T., M. Mohamed, and M. Osman, Investigating the intelligence of the low-tech earth architecture of the Sahara: A feasibility study from the western desert of Egypt. Intelligent Buildings International, 2010. 2(3): p. 179-197.

.8 Elzeyadi, I., A Tale of Two Houses: Environmental Quality, Sustainability, and Indoor Comfort Inside Hassan Fathy's Mit Rehan And A Contemporary Villa in Cairo, Egypt. 1998, The Center for Environmental Design, University of California: Berkeley.

.9 Gado, T. and M. Osman, Investigating traditional natural ventilation methods used in El-Dakhla Oasis, old village. Intelligent Buildings International, 2010. 2(4): p. 267-285.

.10 Elzeyadi, I.M.K., Ten palaces tell their stories: Environmental quality assessment of offices inside 
Vol.41, No. 1. January 2022

School of Architecture. 2009, University of Dundee: Dundee - Uk.

.21 Hammad, H., M. Abdelkader, and A.A. Faggal, Investigating the thermal comfort conditions in an existing school building in Egypt. Journal of Engineering Sciences, Assiut University, Faculty of Engineering, 2017. 45(3): p. 344-359.

.22 Mostafa, A.M., Climatic evaluation of semi-attached residential units: A case study of units in new towns in Egypt, in AEE "Architecture, Energy \& Environment" Tools for climatic design - Advanced International Training Programme. 2001, Lund University: Lund Sweden.

.23 Abdallah, A.S.H., Analysis of Thermal Comfort and Energy Consumption in Long Time Large Educational Halls (Studios), Assiut University, Egypt. Procedia Engineering, 2015. 121: p. 1674-1681.

.24 The Egyptian organization for energy conversation and planning, Architecture and energy manual, EOECP, Editor. 1998: Cairo.

.25 Gado, T. and S. Hosny, Impact of climatic conditions on the thermal comfort in six Egyptian climatic design regions. . Scientific Bulletin of the Faculty of Engineering, Ain Shams University, 2004: p. 100-125.

.26 Szokolay, S.V., Thermal design of buildings. 1987, Canberra: RAIA Education Division.

.27 Cohen, L., L. Manion, and K. Morrison, Research methods in education. 2000, London :Routledge Falmer.

.28 Harry N. Boone, J. and D.A. Boone, Analyzing Likert Data. Journal of extension, 2012. 50.(2)

.29 Crawford, I.M., Marketing research and information systems. Marketing and agribusines texts. Vol. 4. 1997, Rome, Italy: Food and agriculture organization of the united nations.

.30 Osman, M.M.A., U.H. Issa, and A.M.Z. Eraqi, Identifying the risk impact on cost and time of the Egyptian nonresidential buildings projects. International Journal of Scientific Research in Science, Engineering and Technology, 2020. 7(1): p. 1-12. 